\title{
C2D Spitzer-IRS spectra of disks around T Tauri stars
}

\section{PAH emission features ${ }^{\star}$}

\author{
V. C. Geers ${ }^{1}$, J.-C. Augereau ${ }^{1,2}$, K. M. Pontoppidan ${ }^{1,3}$, C. P. Dullemond ${ }^{4}$, R. Visser ${ }^{1}$, J. E. Kessler-Silacci ${ }^{5}$, \\ N. J. Evans $\mathrm{II}^{5}$, E. F. van Dishoeck ${ }^{1}$, G. A. Blake ${ }^{3}$, A. C. A. Boogert ${ }^{6}$, J. M. Brown ${ }^{3}$, F. Lahuis ${ }^{1,7}$, and B. Merín ${ }^{1}$ \\ 1 Leiden Observatory, Leiden University, PO Box 9513, 2300 RA Leiden, The Netherlands \\ e-mail: vcgeers@strw.leidenuniv.nl \\ 2 Laboratoire d'Astrophysique de l'Observatoire de Grenoble, BP 53, 38041 Grenoble Cedex 9, France \\ 3 Division of GPS, Mail Code 150-21, California Institute of Technology, Pasadena, CA 91125, USA \\ 4 Max-Plank-Institut für Astronomie, Koenigstuhl 17, 69117 Heidelberg, Germany \\ 5 Department of Astronomy, University of Texas, 1 University Station C1400, Austin, TX 78712-0259, USA \\ ${ }^{6}$ Division of PMA, Mail Code 105-24, California Institute of Technology, Pasadena, CA 91125, USA \\ SRON, PO Box 800, 9700 AV Groningen, The Netherlands
}

Received 9 January 2006 / Accepted 22 August 2006

\section{ABSTRACT}

\begin{abstract}
Aims. We search for Polycyclic Aromatic Hydrocarbon (PAH) features towards young low-mass (T Tauri) stars and compare them with surveys of intermediate mass (Herbig $\mathrm{Ae} / \mathrm{Be}$ ) stars. The presence and strength of the PAH features are interpreted with disk radiative transfer models exploring the PAH feature dependence on the incident UV radiation, PAH abundance and disk parameters. Methods. Spitzer Space Telescope 5-35 $\mu$ m spectra of 54 pre-main sequence stars with disks were obtained, consisting of $38 \mathrm{~T}$ Tauri, 7 Herbig Ae/Be and 9 stars with unknown spectral type.

Results. Compact PAH emission is detected towards at least 8 sources of which 5 are Herbig Ae/Be stars. The $11.2 \mu$ m PAH feature is detected in all of these sources, as is the $6.2 \mu \mathrm{m}$ PAH feature for the 4 sources for which short wavelength data are available. However, the 7.7 and $8.6 \mu \mathrm{m}$ features appear strongly in only 1 of these 4 sources. Based on the $11.2 \mu \mathrm{m}$ feature, PAH emission is observed towards at least $3 \mathrm{~T}$ Tauri stars, with 14 tentative detections, resulting in a lower limit to the PAH detection rate of $8 \%$. The lowest mass source with PAH emission in our sample is T Cha with a spectral type G8. All 4 sources in our sample with evidence for dust holes in their inner disk show PAH emission, increasing the feature/continuum ratio. Typical $11.2 \mu \mathrm{m}$ line intensities are an order of magnitude lower than those observed for the more massive Herbig Ae/Be stars. Measured line fluxes indicate PAH abundances that are factors of 10-100 lower than standard interstellar values. Conversely, PAH features from disks exposed to stars with $T_{\text {eff }} \leq 4200 \mathrm{~K}$ without enhanced UV are predicted to be below the current detection limit, even for high PAH abundances. Disk modeling shows that the 6.2 and $11.2 \mu \mathrm{m}$ features are the best PAH tracers for T Tauri stars, whereas the 7.7 and $8.6 \mu \mathrm{m}$ bands have low feature over continuum ratios due to the strongly rising silicate emission.
\end{abstract}

Key words. stars: pre-main sequence - planetary systems: protoplanetary disks - circumstellar matter - astrochemistry ISM: molecules

\section{Introduction}

Polycyclic Aromatic Hydrocarbons (PAHs) have been observed in a wide variety of sources in our own and external galaxies. Within the Milky Way PAHs are observed in the diffuse medium, dense molecular clouds, circumstellar envelopes, and (proto-)planetary nebulae (see Peeters et al. 2004 for a summary). A common characteristic of all of these sources is that they are exposed to copious ultraviolet (UV) photons. The UV radiation drives the molecules into excited electronic states, which subsequently decay to lower electronic states through a non-radiative process called internal conversion, followed by vibrational emission in the available $\mathrm{C}-\mathrm{H}$ and $\mathrm{C}-\mathrm{C}$ stretching and bending vibrational modes at 3.3, 6.2, 7.7, 8.6, 11.2, 12.8 and $16.4 \mu \mathrm{m}$. Thus, PAH molecules form an important diagnostic of UV radiation.

$\star$ Appendices $\mathrm{A}$ and $\mathrm{B}$ are only available in electronic form at http://www . aanda.org
In recent years, PAH emission has also been detected from disks around young stars in ground-based and Infrared Space Observatory (ISO) spectra (Van Kerckhoven et al. 2000; Hony et al. 2001; Peeters et al. 2002; van Boekel et al. 2004; Przygodda et al. 2003; Acke \& van den Ancker 2004). The PAH emission is thought to originate from the surface layer of a (flaring) disk exposed to radiation from the central star (cf. models by Manske \& Henning 1999; Habart et al. 2004, hereafter H04). Indeed, ground-based spatially resolved observations show that the features come from regions with sizes typical of that of a circumstellar disk (radius $<12 \mathrm{AU}$ at $3.3 \mu \mathrm{m},<100 \mathrm{AU}$ at $11.2 \mu \mathrm{m}$ ) (Geers et al. 2005; van Boekel et al. 2004; Habart et al. 2005). Searches for PAHs in disks are important because in addition to being a tracer of the strength of the stellar radiation field and disk geometry, the PAHs also affect the disk structure and chemistry. For example, the high opacity of PAHs at FUV wavelengths (Mattioda et al. 2005) could significantly reduce the stellar UV radiation in the inner disk while photoionization of PAHs produces energetic electrons which are a major heating mechanism 
for the gas in the upper layers of the disk where the gas and dust temperatures are not well coupled (Jonkheid et al. 2004; Kamp \& Dullemond 2004).

Detections of PAH features also provide diagnostics of the presence of small grains in the surface layers of disks and the dust evolution through grain growth and dust settling. Evidence for grain growth has been found from modeling of the silicate features from Herbig Ae/Be (hereafter HAeBe) (van Boekel et al. 2003) and T Tauri disks (Przygodda et al. 2003; KesslerSilacci et al. 2005, 2006) and more indirectly from the modeling of the observed $\mathrm{H}_{2}$ emission features from $\mathrm{T}$ Tauri disks (Bergin et al. 2004). PAHs are considered to be on the small end of the size distribution of grains. An important question is whether PAHs have a different timescale for settling and/or growth compared to that of larger silicate/carbon dust grains.

So far, most data obtained on PAHs refer to relatively bright features in the spectra of intermediate mass HAeBe stars. A recent ISO spectroscopic survey has detected PAH features, in particular the most frequently observed $6.2 \mu \mathrm{m}$ feature, towards $57 \%$ of a sample of $46 \mathrm{HAeBe}$ stars (Acke \& van den Ancker 2004). Meeus et al. (2001) classified the ISO observed spectral energy distributions (SEDs) of these intermediate mass young stars into two groups (group I and II). Dominik et al. (2003) interpreted these SEDs in the context of a passive disk model with a puffed-up inner rim (Dullemond et al. 2001) and proposed that group I sources with larger mid-infrared excess have flaring disks while group II sources are consistent with small and/or self-shadowed disks. Acke \& van den Ancker (2004) showed that the group I sources with strong mid-infrared relative to nearinfrared excess display significantly more PAH emission than the group II sources with weaker mid-infrared excesses, consistent with the idea that the PAH emission originates mostly from the disk surface.

The arrival of the Spitzer Space Telescope (Werner et al. 2004) with the InfraRed Spectrograph (IRS) (Houck et al. 2004) provides the opportunity to extend these studies to disks around fainter low mass $T$ Tauri stars. For sources of spectral type $G$ and later, the stellar UV field is orders of magnitude weaker than for HAeBe stars, which will directly affect the PAH excitation and emission. On the other hand, enhanced UV radiation has been detected for some T Tauri stars (e.g., Costa et al. 2000; Bergin et al. 2003). Such an enhanced UV field should be directly reflected in the intensity of the PAH features if these molecules are present in normal abundances. Furthermore, ionized PAHs can be excited by less energetic, optical photons from these cooler stars (Mattioda et al. 2005).

In this paper we present detections of PAH features toward T Tauri stars from our initial set of Spitzer IRS 5-38 $\mu \mathrm{m}$ spectroscopic observations that were taken as part of the Spitzer Legacy program "From Molecular Cores to Planet-Forming Disks" (Evans et al. 2003) (hereafter c2d). The c2d targets consist of a large number of sources with infrared excess in five of the nearest large star-forming regions: Chamaeleon, Lupus, Ophiuchus, Perseus and Serpens. In Paper I, the silicate 10 and $20 \mu \mathrm{m}$ features from pre-main sequence stars with disks are presented and analyzed (Kessler-Silacci et al. 2006). The data are used here to search for PAH features and address a number of outstanding questions. For how many low mass stars can PAH features be found and how does this compare to HAeBe stars? Can limits be put on the abundance of PAHs and thus indirectly on that of the smallest grains? Which factors influence the appearance of PAH features in disks? Can we quantify any additional UV or optical radiation from the strength of the PAH features?
Section 2 describes the sample selection, observations and reduction method. The results for the observed PAH features are presented in Sect. 3 and discussed in Sects. 4 and 5. Conclusions are presented in Sect. 6.

\section{Observations and data reduction}

Mid-infrared spectra were obtained for intermediate and low mass stars with circumstellar disks with the IRS aboard Spitzer as part of the c2d Legacy program. All targets were observed with the Short-High ( $\mathrm{SH})$ module (spectral resolving power of $R \sim 600$ ) covering the wavelength range 10-20 $\mu \mathrm{m}$ and thereby potential PAH features at 11.2 and $12.8 \mu \mathrm{m}$. For a fraction of our sources, Short-Low (SL) observations $(R \sim 100,5-14.5 \mu \mathrm{m})$ were obtained as well, which cover the $6.2,7.7,8.6,11.2$ and $12.8 \mu \mathrm{m}$ PAH features. For the remainder of our sources, the SL observations are in the Guaranteed Time Observation (GTO) IRS program (J.R. Houck) and were at the time of paper submission not yet available.

The original sample selection of the c2d program is described in Evans et al. (2003). In our sample of sources observed to date there are $7 \mathrm{HAeBe}$ stars, $38 \mathrm{~T}$ Tauri stars and 9 sources without known spectral types. These include the $40 \mathrm{~T}$ Tauri stars and 7 HAeBe stars in Kessler-Silacci et al. (2006), although we label 4 of their T Tauri sources here as "unclassified" for lack of spectral type (RNO 15, IRAS 03446+3254, VSSG1, CK 4) and we label 1 of their HAeBe stars here as a T Tauri star since it has spectral type M6 (DL Cha). In addition, we have added the c2d IRS spectra taken for 1 HAeBe star (HD101412), 1 T Tauri star, $\mathrm{Sz} 84$, and 5 sources without known spectral types (EC 69, EC 88, EC 90, EC 92, RNO 91). Our current sample of 54 sources is therefore comparable in size to that of the HAeBe stars observed with ISO. We grouped the sample sources in intermediate and low mass young stars following Thé et al. (1994), as HAeBe stars with spectral types of F7 and earlier, and T Tauri stars with spectral type F8 and later. A summary of the properties of all disk sources can be found in Kessler-Silacci et al. (2006, Paper I) and Merín et al. (in prep.). The sample includes the spectrum of an off-position, taken toward the Ophiuchus cloud at a position devoid of infrared sources, to compare with a typical interstellar medium PAH spectrum.

All spectra were extracted from the SSC pipeline version S12.0.2 BCD images, using the c2d reduction pipeline (KesslerSilacci et al. 2006 and Lahuis, in preparation). The processing includes bad-pixel correction through interpolation using a source profile fit in the cross-dispersion direction. The source profile fitting also gives an estimate of the local sky contribution in the high-resolution spectra. The extracted spectra are defringed using the IRSFRINGE package developed by the $\mathrm{c} 2 \mathrm{~d}$ team and individual orders are in some cases corrected by small scaling corrections $(\$ 5 \%)$ to match the order with the shortest wavelength. Sky subtraction is applied and in a few cases the SH module is scaled down $(\lessgtr 5 \%)$ in flux to match the SL spectrum.

\section{T Tauri stars with PAH features}

\subsection{Identification of $P A H$ features}

Among the 54 disk sources observed to date, a relatively small sample of 8 sources shows one or more clear emission feature(s) that we attribute to PAHs (Fig. 1). Table 1 summarizes the characteristics of these sources. They consist of 3 T Tauri stars and 5 HAeBe stars. Besides the PAH features, some sources show 
Table 1. Sources with potential PAH emission and their characteristics.

\begin{tabular}{|c|c|c|c|c|c|c|c|c|}
\hline Name & RA (J2000) & Dec $(\mathrm{J} 2000)$ & AOR key & Modules $^{a}$ & Dist. [pc] & Sp. type & $\mathrm{H} \alpha(\AA)$ & Ref \\
\hline $\mathrm{LkH} \alpha 330$ & 034548.3 & +322412 & 0005634816 & SL LL1 SH LH & 250 & $\mathrm{G} 3 \mathrm{e}^{e}$ & $11.4^{b}-20.3$ & $3 ; 4,5 ; 4,5$ \\
\hline RR Tau & 053930.5 & +262227 & 0005638400 & SL LL1 SH LH & 160 & $\mathrm{~A} 0 \mathrm{e}-\mathrm{A} 0 \mathrm{IVe}^{e}$ & 21.2 & $11 ; 12,13 ; 5$ \\
\hline HD 98922 & 112231.7 & -532212 & 0005640704 & SH LH & $>540^{f}$ & B9Ve $e^{e}$ & 27.9 & $1 ; 17 ; 10$ \\
\hline HD 101412 & 113944.5 & -601028 & 0005640960 & SL SH LH & 160 & $\mathrm{~B} 9.5 \mathrm{Ve}^{e}$ & 20.4 & $10 ; 16 ; 10$ \\
\hline T Cha & 115713.5 & -792132 & 0005641216 & SH LH & $66_{12}^{+19}$ & G8 $\mathrm{e}^{e}$ & $2-10$ & $1 ; 2 ; 2,18$ \\
\hline HD 135344 & 151548.4 & -370916 & 0005657088 & SH LH & 140 & $\mathrm{~F} 4 \mathrm{Ve}$ & 17.4 & $8 ; 9 ; 10$ \\
\hline $\mathrm{EM}^{*} \mathrm{SR} 21 \mathrm{~N}$ & 162710.3 & -241913 & 0005647616 & SH LH & 125 & $\mathrm{G} 2.5$ & 0.54 & $6 ; 7 ; 19$ \\
\hline VV Ser & 182847.9 & +000840 & 0005651200 & SL SH LH & 259 & $\mathrm{~A} 0 \mathrm{Ve}$ & $22-51^{c}-81.3$ & $14 ; 13 ; 15,4,10$ \\
\hline Off-position & 162400 & -240000 & 0005654272 & SH LH & - & - & - & - \\
\hline VSSG $1^{d}$ & 162618.9 & -242820 & 0005647616 & SH LH & 125 & $\ldots$ & $\ldots$ & $6 ;-;-$ \\
\hline Haro $1-17^{d}$ & 163221.9 & -244215 & 0011827712 & SL LL1 SH LH & 125 & $\mathrm{M} 2.5 \mathrm{e}^{e}$ & 15 & $6 ; 2 ; 2$ \\
\hline
\end{tabular}

${ }^{a} \mathrm{SL}=\mathrm{SL} 1+\mathrm{SL} 2 ;{ }^{b}$ average from 3 measurements; ${ }^{c}$ average from 19 measurements; ${ }^{d}$ PAH feature detected in background spectrum, not associated with the source; ${ }^{e}$ label 'e' added to spectral type here, based on $\mathrm{H} \alpha>0$, not taken from reference; ${ }^{f}$ distance uncertain.

References for distance; spectral type; $\mathrm{H} \alpha$ : 1: van den Ancker et al. (1998), 2: Alcalá et al. (1993), 3: Enoch et al. (2005), 4: Fernandez et al. (1995), 5: Cohen \& Kuhi (1979), 6: Assumed distance to Oph cloud (de Geus et al. 1989), 7: Prato et al. (2003), 8: Acke \& van den Ancker (2004), 9: Dunkin et al. (1997), 10: Acke et al. (2005), 11: Assumed distance to Taurus-Auriga cloud (Kenyon et al. 1994), 12: Hernández et al. (2004), 13: Mora et al. (2001), 14: Straizys et al. (1996), 15: Finkenzeller \& Mundt (1984), 16: Thé et al. (1994), 17: Houk (1978), 18: Alcala et al. (1995), 19: Martin et al. (1998).

Note: sources in the bottom portion of the Table have spectra with PAH features that are fully attributed to background emission.

the $\mathrm{H}_{2} \mathrm{~S}(2)$ line, whereas the $\left[\mathrm{N}_{\mathrm{e}} \mathrm{II}\right] 12.8 \mu \mathrm{m}$ line is detected toward $\mathrm{T}$ Cha. Unlabelled narrow features are likely spurious.

Disk sources with PAH emission features were identified as follows. When both SL and SH spectra are available, we required detection of both a clear $11.2 \mu \mathrm{m}$ feature and a clear $6.2,7.7$ and/or $8.6 \mu \mathrm{m}$ feature. For sources where only a SH spectrum is available $(\lambda>10 \mu \mathrm{m})$, the identification is more critical and largely relies on both the presence of a clear feature at $11.2 \mu \mathrm{m}$ and its shape. We compared potential $11.2 \mu \mathrm{m}$ features with that observed in the off-position spectrum and selected the sources with the lowest residuals, see Sect. 4.3.

This method can introduce a bias against sources with mixed crystalline silicate and PAH features, because crystalline forsterite has a characteristic feature at almost the same central wavelength as the $11.2 \mu \mathrm{m}$ PAH feature. To distinguish between these two possible assignments, we searched for other crystalline silicate features at wavelengths longer than $11.2 \mu \mathrm{m}$ (e.g. 16.2, $18.9,23.7$ and $33.6 \mu \mathrm{m})$. The large wavelength coverage out to $35 \mu \mathrm{m}$ is a significant advantage compared with ground-based data. Three of the five HAeBe and 2 of the $3 \mathrm{~T}$ Tauri sources show (tentative) evidence for crystalline silicates at longer wavelengths, indicated in Table 2 (Kessler-Silacci et al. 2006). In these sources, a contribution from crystalline silicates to the observed 11.2-11.3 $\mu \mathrm{m}$ feature cannot be excluded. The sources illustrate well the difficulties inherent in the identification of PAH emission features at $11.2 \mu \mathrm{m}$ when lacking any information on the presence/absence of other characteristic PAH features at shorter wavelengths. We note also that the $11.2 \mu \mathrm{m}$ PAH feature can be blended with the broad amorphous silicate feature whose strength and spectral width vary with grain size. This may have led us to miss sources with weak $11.2 \mu \mathrm{m}$ emission from PAHs (see discussion in Sect. 3.3), although the characteristic shape of the $11.2 \mu \mathrm{m}$ PAH feature can help in distinguishing between PAHs and silicates, as discussed in Sect. 4.3.

Finally, sources with PAH features but showing silicate and/or ice features in absorption have been excluded from the sample presented here. For comparison, Fig. 2 includes observations of the off-source spectrum and of two more late type sources, Haro 1-17 and VSSG1 which were selected on the above mentioned criteria but for which background extraction

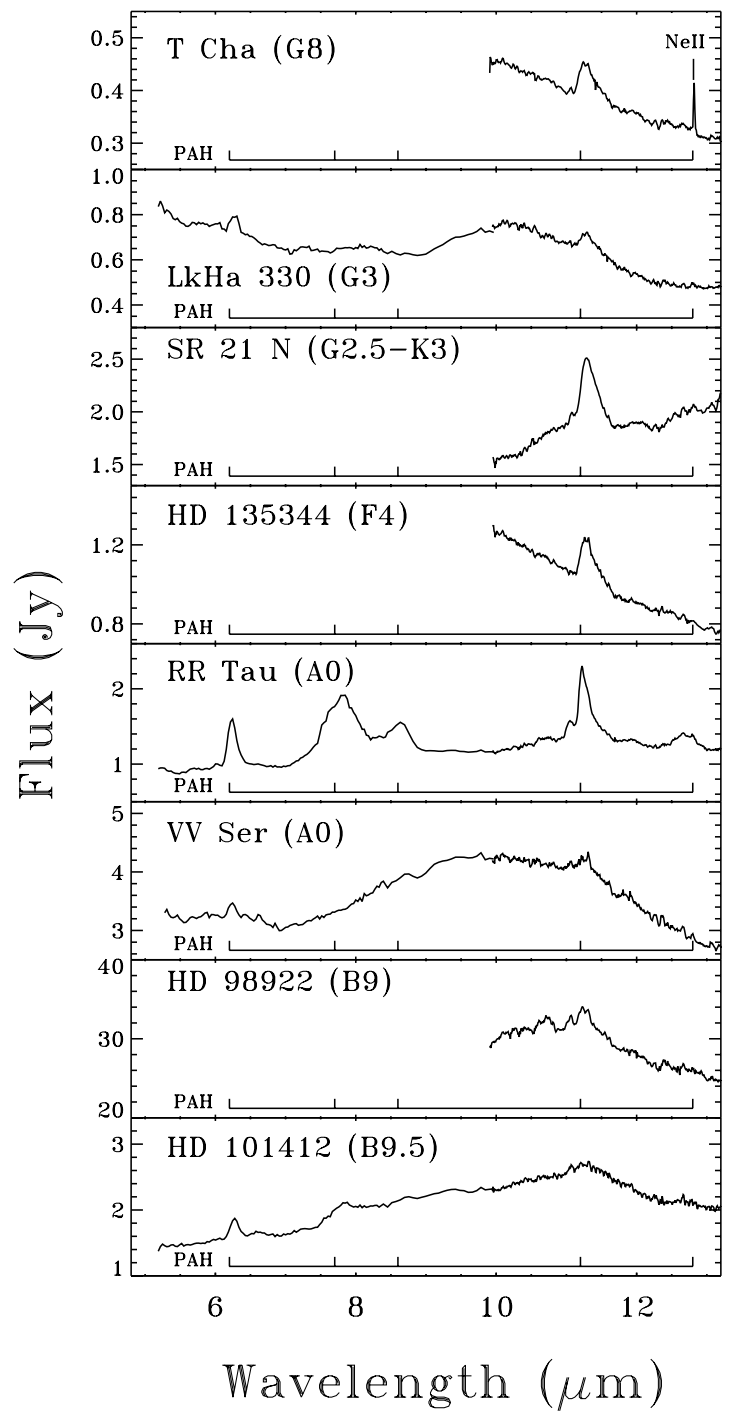

Fig. 1. Spitzer IRS spectra of sources with PAH features, comprised of the SL $(5-10 \mu \mathrm{m})$ and SH $(10-20 \mu \mathrm{m})$ modules. The location of PAH features is indicated with markers at 6.2, 7.7, 8.6, 11.2 and $12.8 \mu \mathrm{m}$. 


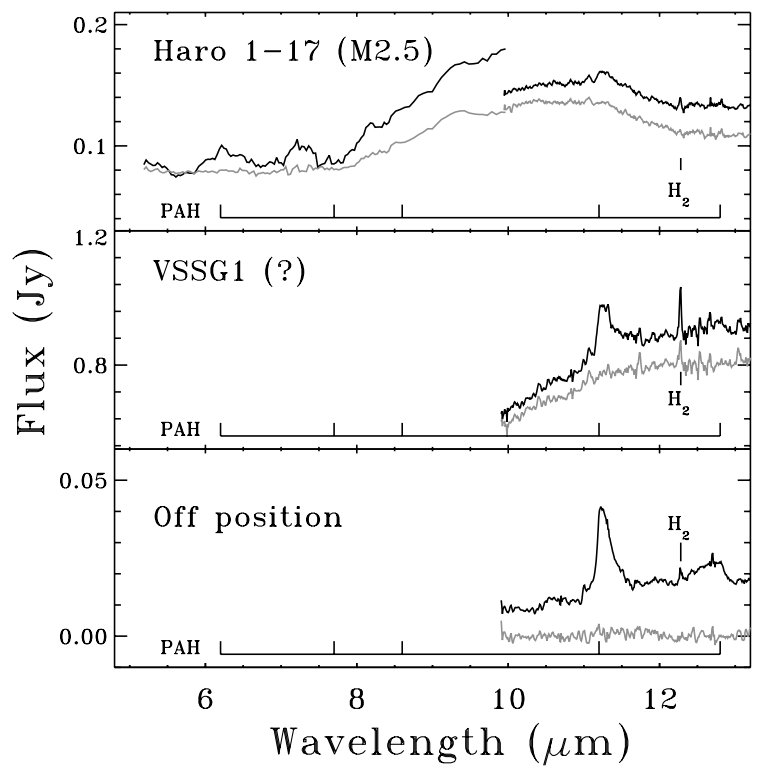

Fig. 2. Spitzer IRS spectra (black line) of sources with PAH features that are associated with extented cloud emission, comprised of the SL (5$10 \mu \mathrm{m})$ and $\mathrm{SH}(10-20 \mu \mathrm{m})$ modules. The extended emission corrected spectrum is shown in grey. The location of PAH features is indicated with markers at $6.2,7.7,8.6,11.2$ and $12.8 \mu \mathrm{m}$.

shows that the PAH emission features are fully associated with the extended cloud emission (see below).

\subsection{Spatial extent of $P A H$ emission}

An important question is whether the PAH emission originates primarily from the star+disk system or from an extended nebulosity around the star. To properly answer this question, either spatially resolved spectra or images in specific PAH band filters (both at feature wavelength and slightly off-peak for determination of strength and extent of continuum emission) with sufficient (subarcsec) spatial resolution are required. Since such data are lacking for our sample we use the extracted background and long-slit spectra to provide constraints on the extent of the emission.

The SL spectra are taken using long-slit spectroscopy and the 2D spectral images can be used to determine if the features are seen extended along the entire width of the slit. An example 2D spectral image of module SL is shown for RR Tau in Fig. 3, where no extended emission is seen along the slit. The pixel size of the SL module is $1.8^{\prime \prime}$, which means that any feature originating from a region smaller than 2 pixels (e.g. $540 \mathrm{AU}$ at $150 \mathrm{pc}$ ) will be spatially unresolved. A similar lack of extended emission in SL1 is seen for all of our PAH sources with the exception of Haro 1-17. Thus, the PAH emission for these sources is constrained to originate from a region of at most 3.6".

The SL spectra presented have been corrected for the estimated background contribution (see Sect.2). In 4 of the $5 \mathrm{SL}$ spectra (LkH $\alpha$ 330, RR Tau, HD 101412 and VV Ser), the features remain after background subtraction and are concluded to be associated with the source. However, for 1 source, Haro 117, the sky-corrected SL spectrum shows no PAH features (see Fig. 2). The PAH emission seen towards this source is concluded to be entirely due to background emission.

A number of the sources were only observed in $\mathrm{SH}$ for which the width of the slit is too small to directly extract the continuum flux outside the source profile. Here, the background
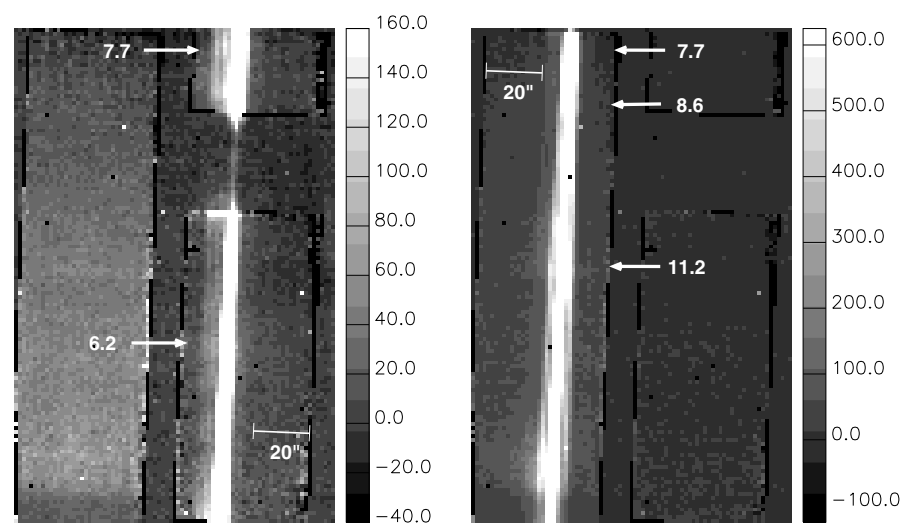

Fig. 3. 2D spectral image of modules SL2 (left panel) and SL1 (right panel) for RR Tau. The quantity shown is the ADU in units of $\mathrm{e}^{-} \mathrm{s}^{-1}$. The positions of the 6.2, 7.7, 8.6 and 11.2 $\mu \mathrm{m}$ PAH features are indicated with labels. The horizontal bar at bottom right of the left panel and top left of the right panel indicates a spatial extent of $20^{\prime \prime}$.

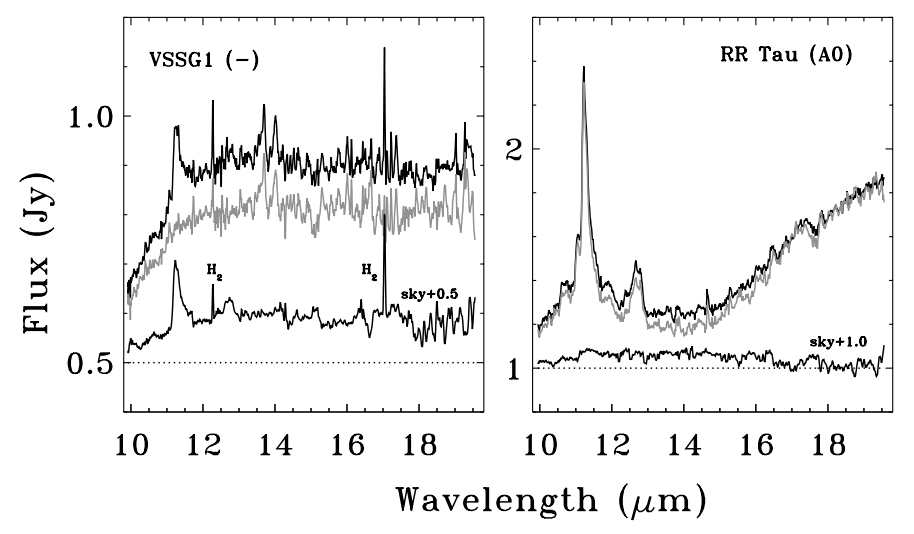

Fig. 4. IRS SH spectra for VSSG 1 and RR Tau. Bottom (black): extracted sky spectrum. Middle (grey): source spectra corrected for background. Top (black): source spectra. The sky spectra are shifted by +0.5 and +1.0 Jy respectively for purpose of clarity.

contribution is estimated from fitting a standard star source profile plus a flat continuum flux to the measured source profile. This background subtraction is more difficult, since the small slit length of only 11.2" (5 pixels) makes the source profile fits less accurate.

For all presented sources, the SH source and sky spectra were inspected for PAH features. Two SH background spectra, one with extended PAH features (VSSG1) and one without (RR Tau) are shown in Fig. 4. The extracted background spectra for $\mathrm{SH}$ show no PAH features for 8 of the 10 sources in our sample with PAH emission. The two sources with features in the background spectra are Haro 1-17 and VSSG1. In both cases, removing the background emission entirely removes the $11.2 \mu \mathrm{m}$ PAH feature from the source spectrum (Fig. 2). It is concluded that for these two sources the PAH features are fully due to background emission.

The environment around one source, the Herbig Ae star VV Ser, is discussed extensively by Pontoppidan et al. (2006, submitted). Based on IRAC and MIPS images, it is shown that VV Ser is surrounded by a bright and extremely large $\left(\sim 6^{\prime}\right)$ nebulosity emitting at 8,24 and $70 \mu \mathrm{m}$, which is not seen in near infrared images. They conclude that the emission is due to a mix of quantum-heated PAHs and Very Small Grains (VSGs) present in the low-density nebula. The PAH emission lines in the spectrum 
must originate from within $1.8^{\prime \prime}-2.35^{\prime \prime}$ or 450-600 AU (halfslit width SL-SH). In their best-fit model they require a central cavity of $15000 \mathrm{AU}$, so the extended nebulosity is not expected to account for the observed PAH features although they note that it cannot be ruled out that a small clump of PAH material is present nearer to the star. Here we assume the emission is from PAHs in the circumstellar disk.

In summary, we conclude that the PAH emission from most of our sources does not originate from extended diffuse fore- or background emission and must instead originate from the observed young stars with disks.

\subsection{Statistics}

Within our current c2d sample, clear PAH features are detected in most (5 out of 7) HAeBe stars while only 3 out of $38 \mathrm{~T}$ Tauri stars show features consistent with the presence of PAH molecules. Interestingly, the PAH detection rate is $100 \%$ for the 4 sources (HD135344, SR 21N, LkH $\alpha 330$ and T Cha) with SEDs characteristic of cold disks, i.e., sources with SEDs that lack excess emission in the 3-13 $\mu \mathrm{m}$ region, indicative of an inner hole in the dust disk (Brown et al., in prep). These 4 sources are also the 4 lowest mass sources (spectral type F4 - G8) with PAH detections.

The $\mathrm{c} 2 \mathrm{~d}$ sample of PAH detections is biased towards sources with either a strong $11.2 \mu \mathrm{m}$ feature or multiple PAH features from $\mathrm{SH}$ and SL observations. This excludes potential sources with weak $\mathrm{PAH}$ features for which only $\mathrm{SH}$ observations are available now: since we cannot assign the origin of this $11.2 \mu \mathrm{m}$ feature to either PAH or crystalline forsterite, these sources are, for now, excluded. This includes 17 sources with tentative 11.2 $\mu$ m detections: $14 \mathrm{~T}$ Tauri stars, 1 Herbig Ae star and 2 unclassified sources. The $14 \mathrm{~T}$ Tauri stars are DoAr 24E, EC 82, GW Lup, GY 23, HT Lup, Krautter's Star, RU Lup, SX Cha, SY Cha, SZ 73, VW Cha, VZ Cha, V710 Tau (binary) and WX Cha. Future SL data will be able to confirm or dismiss the presence of PAHs in these sources.

Finally, in the current sample there are 28 sources with no clear $11.2 \mu \mathrm{m}$ PAH feature, consisting of 1 Herbig Ae star, $21 \mathrm{~T}$ Tauri stars and 6 sources with no known spectral type. From our present sample, the lower limit to the detection rate of $\mathrm{PAH}$ emission features toward T Tauri stars is about $8 \%$. This detection rate goes up to $45 \%$ if tentative detections are included. PAH emission is only detected toward the 3 more massive T Tauri stars of spectral type $\mathrm{G}$ of our sample, which consists mostly of $\mathrm{K}-\mathrm{M}$ type stars.

The rather small fraction (8\%) of low mass stars in our c2d sample with PAH emission features, based on the $11.2 \mu \mathrm{m}$ PAH feature, contrasts with the large fraction $(57 \%$ according to Acke \& van den Ancker 2004) for intermediate mass stars detected with ISO based on the $6.2 \mu \mathrm{m}$ PAH feature. If only the $11.2 \mu \mathrm{m}$ features are considered, their detection rate drops to $48 \%$ based on their Table 3 . However, this potentially includes sources with only crystalline silicate emission.

\section{Analysis of PAH features}

\subsection{Overview of detected PAH features}

Table 2 summarizes the detected PAH features and measured line fluxes. The spectrum of RR Tau nicely shows all the main PAH bands detectable in the IRS spectral window at 6.2, 7.7, 8.6, 11.2, $12.8 \mu \mathrm{m}$ (Fig. 1) and even $16.4 \mu \mathrm{m}$ (not shown). In contrast with the other sources, it has clear 7.7 and $8.6 \mu \mathrm{m}$ features,
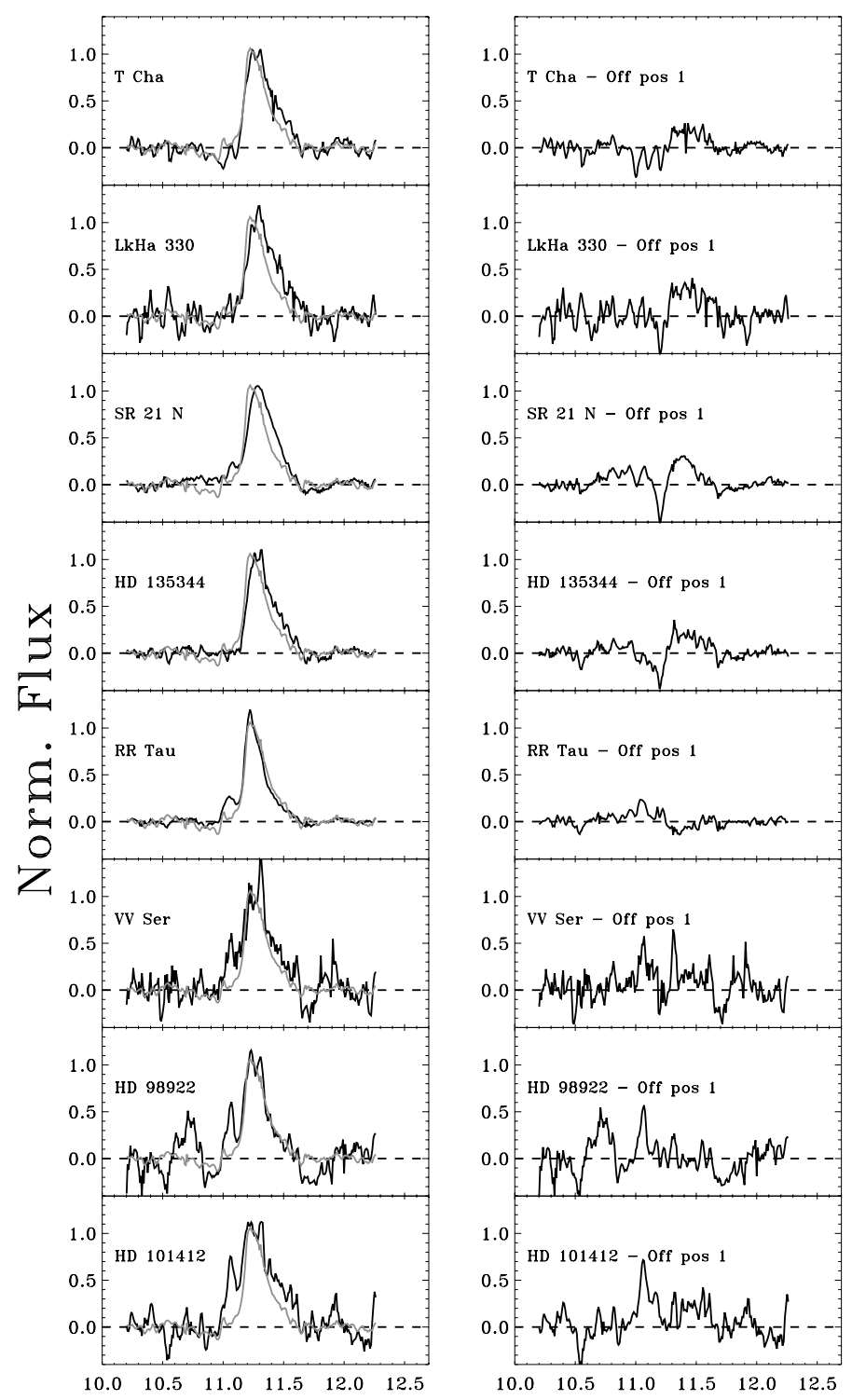

\section{Wavelength $(\mu \mathrm{m})$}

Fig. 5. Left panel: blow-up of the SH spectra around the continuumsubtracted $11.2 \mu \mathrm{m}$ PAH feature, normalized to the fitted peak flux (black line). Overplotted in light grey for comparison is the SH spectrum of the off-position. Right panel: plot of the difference between the source and the off-position spectra.

attributed to $\mathrm{C}-\mathrm{C}$ stretching transitions and $\mathrm{C}-\mathrm{H}$ in plane bending transitions. These features are either less obviously detected above the silicate continuum or simply absent in the 4 other sources with SL spectra. $\mathrm{LkH} \alpha 330$ is a clear example of a spectrum with a $6.2 \mu \mathrm{m} \mathrm{C}-\mathrm{C}$ stretching feature and a $11.2 \mu \mathrm{m} \mathrm{C}-\mathrm{H}$ out-of-plane bending feature but no $7.7,8.6$ nor $12.8 \mu \mathrm{m}$ features. The 7.7 and $8.6 \mu \mathrm{m}$ features are generally found to be well correlated with the $6.2 \mu \mathrm{m}$ feature (Peeters et al. 2002) and their absence is thus puzzling in our high sensitivity spectra. Spoon et al. (2002) show that silicate absorption can strongly mask the $8.6 \mu \mathrm{m}$ PAH feature; in our case the silicate is in emission, however. According to Acke \& van den Ancker (2004) (see their Table 3), four HAeBe stars observed with ISO similarly show 6.2 and $11.2 \mu \mathrm{m}$ emission but no features at 7.7 or $8.6 \mu \mathrm{m}$. Two of these sources, HD 163296 and VV Ser, are also in the c2d sample (the other two are HD 142666 and HD 144432). This absence 
Table 2. Line fluxes in $\mathrm{W} \mathrm{m}^{-2}$ and feature/continuum ratio of PAH features.

\begin{tabular}{|c|c|c|c|c|c|c|c|}
\hline \multirow[b]{2}{*}{ Name } & \multirow[b]{2}{*}{$\begin{array}{l}6.2 \mu \mathrm{m} \\
(\mathrm{SL})\end{array}$} & \multicolumn{2}{|c|}{ Line flux } & \multicolumn{4}{|c|}{ Feature/continuum } \\
\hline & & $\begin{array}{l}11.2 \mu \mathrm{m} \\
(\mathrm{SL})\end{array}$ & $\begin{array}{c}11.2 \mu \mathrm{m} \\
(\mathrm{SH})\end{array}$ & $\begin{array}{c}12.8 \mu \mathrm{m} \\
(\mathrm{SH})\end{array}$ & $\begin{array}{c}6.2 \mu \mathrm{m} \\
(\mathrm{SL})\end{array}$ & $\begin{array}{c}11.2 \mu \mathrm{m} \\
(\mathrm{SH})\end{array}$ & $\begin{array}{l}\text { cryst. } \\
\text { sil. }^{c}\end{array}$ \\
\hline $\mathrm{LkH} \alpha 330$ & $1.7 \times 10^{-15}$ & $7.7 \times 10^{-16}$ & $5.1 \times 10^{-16}$ & $\leq 2.5 \times 10^{-16}$ & 1.21 & 1.12 & $\mathrm{~T}$ \\
\hline RR-Tau & $9.6 \times 10^{-15}$ & $5.0 \times 10^{-15}$ & $4.5 \times 10^{-15}$ & $1.3 \times 10^{-15}$ & 1.71 & 1.57 & $\mathrm{Y}$ \\
\hline HD 98922 & $-a$ & $-a$ & $1.4 \times 10^{-14}$ & $4.0 \times 10^{-15}$ & - & 1.06 & $\mathrm{Y}$ \\
\hline HD 101412 & $4.0 \times 10^{-15}$ & $2.2 \times 10^{-15}$ & $1.5 \times 10^{-15}$ & $3.0 \times 10^{-16}$ & 1.19 & 1.08 & $\mathrm{Y}^{d}$ \\
\hline T Cha & $-^{a}$ & $-^{a}$ & $3.3 \times 10^{-16}$ & $1.1 \times 10^{-16}$ & - & 1.15 & $\mathrm{~N}$ \\
\hline HD 135344 & $-^{a}$ & $-{ }^{a}$ & $1.2 \times 10^{-15}$ & $1.1 \times 10^{-16}$ & - & 1.19 & $\mathrm{~N}$ \\
\hline $\mathrm{EM}^{*} \mathrm{SR} 21 \mathrm{~N}$ & $-^{a}$ & $-^{a}$ & $4.0 \times 10^{-15}$ & $4.1 \times 10^{-16}$ & - & 1.32 & $\mathrm{~T}$ \\
\hline VV Ser & $2.6 \times 10^{-15}$ & $3.1 \times 10^{-15}$ & $2.3 \times 10^{-15}$ & $1.2 \times 10^{-15}$ & 1.07 & 1.07 & $\mathrm{Y}$ \\
\hline Off-position 1 & $-{ }^{a}$ & $-{ }^{a}$ & $1.4 \times 10^{-16}$ & $5.5 \times 10^{-17}$ & - & & - \\
\hline VSSG $1^{b}$ & $-{ }^{a}$ & $-{ }^{a}$ & $8.6 \times 10^{-16}$ & $2.4 \times 10^{-16}$ & - & & $\mathrm{Y}$ \\
\hline Haro $1-17^{b}$ & $4.5 \times 10^{-16}$ & $\leq 2.5 \times 10^{-16}$ & $\leq 2.5 \times 10^{-16}$ & $\leq 2.5 \times 10^{-16}$ & & & $\mathrm{Y}$ \\
\hline
\end{tabular}

${ }^{a}$ No SL spectra available; ${ }^{b}$ PAH feature detected in background spectrum, not associated with the source; ${ }^{c}$ crystalline silicates detected in either 28-29, or 33-35 $\mu \mathrm{m}$, from Kessler-Silacci; et al. 2006, Table 2; "Y" if detected, "N" if not detected, or "T" if the identification is tentative; ${ }^{d}$ derived in this study.

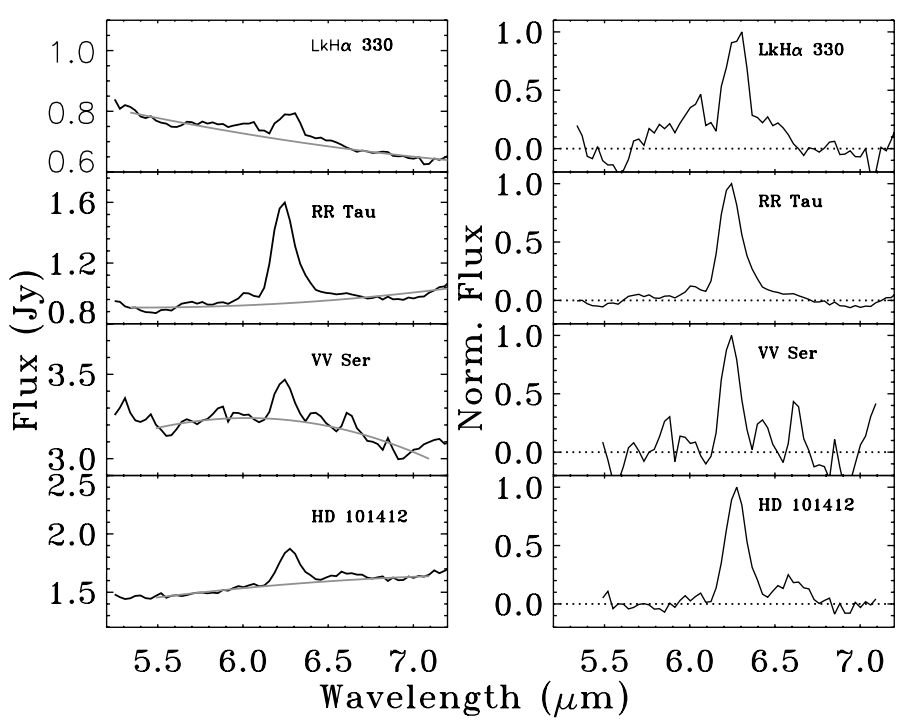

Fig. 6. Left: blow-up of the Spitzer-IRS low resolution spectra around the $6.2 \mu \mathrm{m}$ PAH feature. A simple fit of the continuum flux is plotted with a light solid line. Right: continuum subtracted spectra, normalised to the peak flux of the PAH feature.

is further discussed in Sect. 5.5, in the context of a disk model which demonstrates the lower contrast of the 7.7 and $8.6 \mu \mathrm{m}$ features with respect to the continuum emission from the disk.

\subsection{Line flux determination}

To determine the strength of the PAH features, the continuum emission needs to be subtracted. As a simple approximation we derive a local pseudo-continuum by fitting a $2 \mathrm{D}$ polynomial to the spectrum around the individual PAH features, where the continuum is selected by hand. For the $11.2 \mu \mathrm{m}$ feature, a polynomial is fitted to the emission at $10.5-11.0$ and $11.8-12.2 \mu \mathrm{m}$. The continuum emission below the $6.2 \mu \mathrm{m}$ emission is estimated between 5.5 and $7.1 \mu \mathrm{m}$. We do not include the 7.7 and $8.6 \mu \mathrm{m}$ features in Table 2 because these do not appear significantly in our sources with SL data, with the exception of RR Tau. The $12.8 \mu \mathrm{m}$ line fluxes extracted from SL are within the uncertainty consistent with those extracted from $\mathrm{SH}$.
The continuum-subtracted features are integrated between fixed wavelengths, in particular between 6.0 and $6.6 \mu \mathrm{m}$ for the $6.2 \mu \mathrm{m}$ PAH feature, between 10.9 and $11.6 \mu \mathrm{m}$ for the $11.2 \mu \mathrm{m}$ feature (see Fig. B.1 in the online material) and between 11.8 and $13.2 \mu \mathrm{m}$ for the $12.8 \mu \mathrm{m}$ feature. The resulting continuum subtracted features are shown in Figs. 5, 6 and B.2.

The measured line fluxes are summarized in Table 2. Thanks to the increased sensitivity of Spitzer, our derived line fluxes for clearly detected PAH features are an order of magnitude lower that what was previously possible with ISO. Our weakest detected feature is the $11.2 \mu \mathrm{m}$ PAH feature in T Cha with a line flux of $3.3 \times 10^{-16} \mathrm{~W} \mathrm{~m}^{-2}$. A mean $3 \sigma$ sensitivity limit of $2.5 \times 10^{-16} \mathrm{~W} \mathrm{~m}^{-2}$ is derived from the noise determination in the continuum adjacent to the PAH features, though this limit varies somewhat from source to source, depending on differences in the $\mathrm{S} / \mathrm{N}$ of the reduced spectra and on the presence of residual reduction artifacts for a few cases. For a small number of sources the sensitivity limit reaches a few $\times 10^{-17} \mathrm{~W} \mathrm{~m}^{-2}$.

Three HAeBe stars in our sample - HD 135344, RR Tau and VV Ser - have previously been observed with ISO, albeit with much lower $S / N$ ratio (Acke \& van den Ancker 2004). For RR Tau, our derived line flux for the $6.2 \mu \mathrm{m}$ feature agrees within $\sim 5 \%$ with the ISOPHOT-S spectra, while the $11.2 \mu \mathrm{m}$ feature is larger by about a factor 2 in the IRS spectrum. For HD 135344, we derive a slightly higher $11.2 \mu \mathrm{m}$ line flux than the ISO upper limit. For VV Ser, our derived $6.2 \mu \mathrm{m}$ line flux is about 4 times weaker, whereas our $11.2 \mu \mathrm{m}$ detection is consistent with the ISO data.

Sloan et al. (2005) have presented Spitzer SL observations for 4 HAeBe stars with spectra showing PAH features but no silicate dust features, among which HD 135344 is included in our sample. They report clear 6.2, "7.9", 11.3 and $12.7 \mu$ m features for all of their sources (HD 34282, HD 135344, HD 141569, HD 169142). For HD 135344 the $7.9 \mu \mathrm{m}$ feature is weaker and broader and the $8.6 \mu \mathrm{m}$ feature is absent. Their derived line flux for the $11.2 \mu \mathrm{m}$ PAH feature is consistent with ours within $10 \%$.

\subsection{Comparison of $\mathrm{PAH}$ features}

In a previous study of a diverse sample of interstellar and circumstellar sources, planetary nebulae, reflection nebulae HII regions and galaxies (van Diedenhoven et al. 2004), the $11.2 \mu \mathrm{m}$ 
PAH feature of almost all YSO's, non-isolated HAeBe stars and HII regions was found to have a similar asymmetric profile with a $F W H M$ of $\sim 0.17 \mu \mathrm{m}$, and a peak wavelength in the range of 11.2-11.24 $\mu \mathrm{m}$. The single isolated HAeBe source in their sample, HD 179218, shows a broader 11.2 $\mu \mathrm{m}$ PAH feature with a peak wavelength of $\sim 11.25 \mu \mathrm{m}$. Our peak position lies in the range $11.25-11.32 \mu \mathrm{m} \pm 0.03 \mu \mathrm{m}$, but this determination is influenced by the uncertainty in the continuum fit, and the shift to longer wavelengths may be partially explained by the presence of a $11.3 \mu \mathrm{m}$ feature from crystalline silicates.

Figure 5 shows a comparison of the continuum-subtracted $11.2 \mu \mathrm{m}$ features from all SH spectra. The off-source PAH feature, which is clearly not contaminated by silicate emission, is included. Both the source and the off-source features are normalized to the peak flux. In the right panel of Fig. 5 the difference between the two features is shown.

For T Cha, LkHa 330, SR $21 \mathrm{~N}$ and HD 135344, the $11.2 \mu \mathrm{m}$ feature is broader than, as well as redshifted with respect to, the off-position feature. Of these, T Cha and HD 135344 show no evidence for crystalline silicates at 28-29 and 33.6 $\mu \mathrm{m}$ (KesslerSilacci et al. 2006), while $\mathrm{LkH} \alpha 330$ and SR $21 \mathrm{~N}$ show tentative crystalline features. Thus, the presence of a $11.3 \mu \mathrm{m}$ crystalline silicate feature cannot readily explain the broadening of the measured feature for these sources. Such a broad shape has been seen before in the planetary nebulae IRAS 17047-5650 and IRAS 21282+5050 (Hony et al. 2001). Pech et al. (2002) proposed anharmonicity as an explanation of the broadening and used a PAH emission model to fit the $11.2 \mu \mathrm{m}$ feature of IRAS $21282+5050$ with a combination of the fundamental $(v=1 \rightarrow 0)$ and hot bands ( $v=2 \rightarrow 1$ and $v=3 \rightarrow 2$ ) of the transition. These hot bands would point to very hot PAHs being present, presumably in the innermost part of the disk where the radiation field is strongest.

For the HAeBe sources RR Tau, VV Ser, HD 98922 and HD 101412, the peak wavelength of the 11.2 PAH feature is very similar to that of the off-position feature. For RR Tau, the PAH feature compares very well with the off-source feature, both in shape and peak position, showing little to no residual after subtraction. For the other 3 sources, subtracting the off-position feature leaves several residual features, hinting at the presence of crystalline features, which are also seen at longer wavelengths.

\section{PAH emission from disks}

\subsection{Disk model}

The strength of the PAH emission features is known to depend on the strength of the UV and optical radiation field, but in disks several additional parameters can affect the appearance of the $\mathrm{PAH}$ features. Here we address the question as to how the PAH features are affected by the spectrum of the central source, the PAH abundance and the flaring geometry of the disk. A related question is why no PAH features are seen in at least half of our sample of $\mathrm{T}$ Tauri disks, in contrast to the findings for HAeBe stars.

We use the 3-dimensional Monte Carlo radiative transfer code RADMC (Dullemond \& Dominik 2004), for which a module to treat the emission from quantum-heated PAH molecules and Very Small Grains has been included. This module will be described in detail in Dullemond et al. (in prep.), but a rough description has been given by Pontoppidan et al. (2006, submitted). A template model is set up using the following model parameters. A Kurucz model spectrum is taken for the central star with $T_{\text {eff }}=10000 \mathrm{~K}$, but the stellar parameters (luminosity, stellar radius) were chosen from evolutionary tracks by Siess et al. (2000) for an age of 3 Myr. The disk is modeled with $M_{\text {disk }}=1 \times 10^{-2} M_{\odot}$, an inner radius set by a dust evaporation temperature of $1300 \mathrm{~K}$, and an outer radius of $300 \mathrm{AU}$. The disk is flaring, with the vertical pressure scale height (in units of radius) at the inner $\operatorname{rim} H_{p} / R_{\text {in }}=0.02$ and at the outer edge $H_{p} / R_{\text {out }}=0.14$. The disk is modeled to be close to face-on $\left(i=5.7^{\circ}\right)$, to maximize the strength of the PAH features. The spectra are scaled to a distance to the observer of $150 \mathrm{pc}$.

The PAH emission is calculated for an equal mix of neutral and singly ionized $\mathrm{C}_{100} \mathrm{H}_{24}$ molecules, adopting the Draine \& $\mathrm{Li}$ (2001) PAH emission model, using the "thermal continuous" approximation. Multi-photon events are included for the PAH excitation, following the method outlined by Siebenmorgen et al. (1992). Enhancement factors for the integrated cross sections of the 6.2, 7.7 and $8.6 \mu \mathrm{m}$ bands $\left(E_{6.2}=3, E_{7.7}=2, E_{8.6}=2\right)$ as suggested by Li \& Draine (2001) are taken into account, as also implemented in H04. The actual ionization state of PAH, which varies as a function of location in the disk, can affect the 7.7 and $8.6 \mu \mathrm{m}$ features relative to 11.2 and $3.3 \mu \mathrm{m}$ but this will be explored further in future models which include a full ionization balance of multiple PAH species in disks (Visser et al. in prep). We include the opacities from Mattioda et al. (2005) for near-infrared wavelengths. Several model tests were performed, which are presented in Appendix A, including a comparison with H04.

PAH destruction is expected to occur in a strong UV radiation field, when the PAH molecules, through multi-photon events, absorb more than $21 \mathrm{eV}$ in an interval shorter than their cooling timescale (Guhathakurta \& Draine 1989). If the PAH destruction happens on a shorter timescale than the lifetime of the disk, this can have an effect on the PAH abundance in, and expected PAH emission from, the inner region of the disk. This effect will be stronger for smaller $\left(N_{\mathrm{c}}<50\right)$ PAHs, and depends on the assumed temperature at which the PAHs dissociate. Model calculations by Visser et al. (in prep.) show that for PAHs with $N_{\mathrm{c}}=100$ the lifetime is larger than the lifetime of the disk, and therefore the PAHs are kept at constant abundance throughout the entire the disk. Tests with removing PAHs inwards from a destruction radius $R_{\mathrm{PAH}, \text { in }}$ (by setting their abundance to zero) show that the results do not depend sensitively on the choice of $R_{\mathrm{PAH} \text {,in }}$ when it is of order $1 \mathrm{AU}$.

In our template model, we use the PAH abundance of $\mathrm{H} 04$, who adopt a fraction of $23 \%$ of available carbon being locked up in $\mathrm{C}_{100} \mathrm{H}_{24}$ PAHs, corresponding to a carbon abundance with respect to hydrogen of $5 \times 10^{-5}$. For a single PAH species with $N_{\mathrm{C}}=100\left(\mathrm{C}_{100} \mathrm{H}_{24}\right)$ this leads to an abundance of $5 \times 10^{-7}$. In our model the abundance is set as a fraction of dust mass. Assuming $\mathrm{C}_{100} \mathrm{H}_{24}$ (molecular mass: $2.04 \times 10^{-21} \mathrm{~g}$ ) and a dust-to-gas ratio of 1 to 100 , the PAH abundance of $5 \times 10^{-7}$ with respect to hydrogen corresponds to a mass fraction of 0.061 gram of PAH per gram of dust, of which 50\% ionized and 50\% neutral. Such an abundance is at the high end of that inferred for general interstellar clouds (Cesarsky et al. 2000; Habart et al. 2001). Indeed, the parameters chosen in our model and that of H04 maximize the PAH emission.

\subsection{Dependence on spectral type}

Since UV and optical radiation incident on the disk surface provides the main PAH excitation mechanism, the PAH features must depend on the spectral type of the illuminating star. To address this influence our template disk model is calculated for a range of Kurucz stellar spectra, with $T_{\text {eff }}=10000,8000,6000$, 

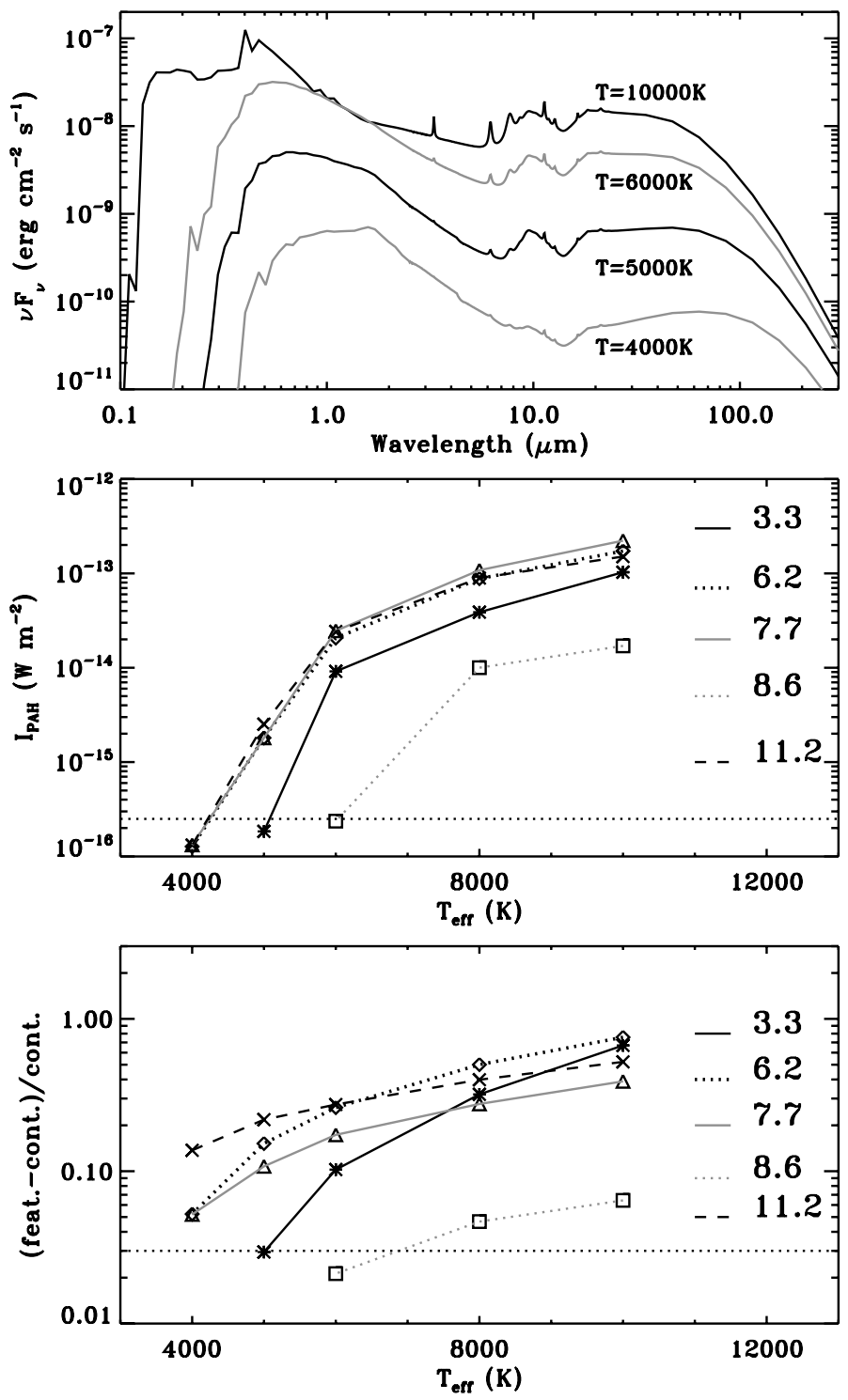

Fig. 7. Top panel: Model SEDs of a star+disk with PAHs, for different spectral types of the star, with $T_{\text {eff }}=10000,6000,5000$ and $4000 \mathrm{~K}$ at a distance of $150 \mathrm{pc}$. Middle panel: PAH line flux of the various features for the corresponding models, including also $T_{\text {eff }}=8000 \mathrm{~K}$. Bottom panel: Feature to continuum ratio of the PAH features. The dotted line indicates our $3 \sigma$ observational limits.

5000 and $4000 \mathrm{~K}$, corresponding to spectral types A0, A6, G0, K2 and K7, respectively (Gray \& Corbally 1994). Stellar parameters are again taken from evolutionary tracks (Siess et al. 2000) for an age of $3 \mathrm{Myr}$. The disk parameters were modified slightly in 1 iteration to ensure hydrostatic equilibrium throughout the disk, except for the inner rim. The model SEDs for the different central stars and absolute continuum subtracted fluxes for the PAH features are presented in Fig. 7. A blowup of the standard model spectrum is shown in Fig. 8.

Since the absolute strength of the PAH features scales foremost with the total radiation that is absorbed by the PAHs, it also depends on disk parameters that are unrelated to the PAHs themselves. To evaluate the role of the disk continuum, we present feature/continuum ratios in Fig. 7 as well.

It is seen that with decreasing $T_{\text {eff }}$ both the line flux as well as the feature/continuum ratio decrease for the 3.3, 6.2, 7.7, 8.6 and $11.2 \mu \mathrm{m}$ features (Fig. 7). The rate of decrease in line flux is similar for all features, as is that of the feature/continuum

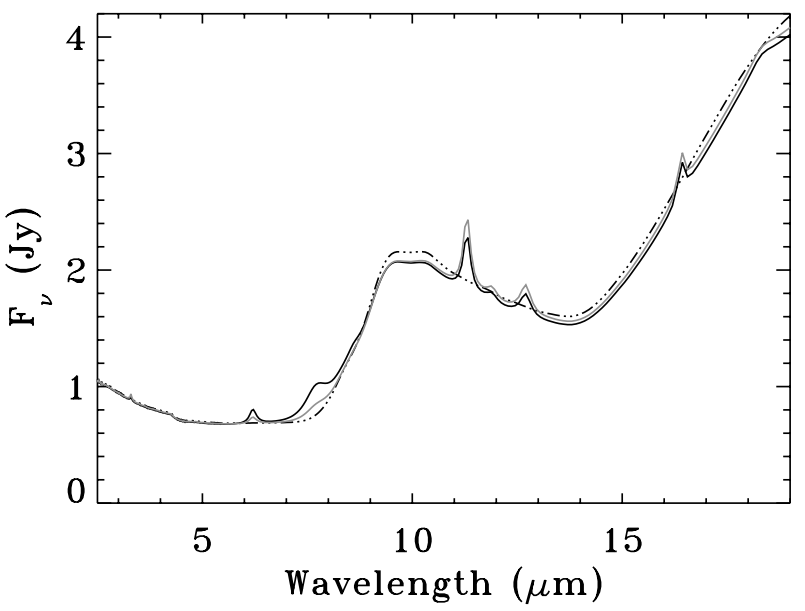

Fig. 8. Blowup of the standard model spectrum of a star+disk with PAHs, for $T_{\text {eff }}=6000 \mathrm{~K}$ with template parameters (dark solid line), without PAHs (dash-dotted line), with only neutral $\mathrm{C}_{100} \mathrm{H}_{24} \mathrm{PAH}$ (light solid line). Note that the 11.2 and $6.2 \mu \mathrm{m}$ features are clearly visible on top of the continuum, whereas the 7.7 and $8.6 \mu \mathrm{m}$ features are masked by the rising $10 \mu \mathrm{m}$ silicate feature.

ratio except for the $3.3 \mu \mathrm{m}$ feature. The latter feature disappears more rapidly with decreasing $T_{\text {eff }}$ because the continuum emission at $3.3 \mu \mathrm{m}$ decreases at a slower rate compared to the longer wavelengths. For all $T_{\text {eff }}$ considered, the $8.6 \mu \mathrm{m}$ feature has the lowest feature to continuum ratio, from $\lesssim 7$ to $\lesssim 3 \%$, which can be clearly seen in Fig. 8. When the PAH molecules are assumed to be $100 \%$ neutral $\mathrm{C}_{100} \mathrm{H}_{24}$, the feature/continuum ratio of the $6.2,7.7$ and $8.6 \mu \mathrm{m}$ bands decreases by a factor $\sim 2-3$, while that of the 3.3 and $11.2 \mu \mathrm{m}$ features increases by a factor $\sim 1.5-3$ (Fig. 8).

These models show that the 6.2 and $11.2 \mu \mathrm{m}$ features are the most suitable tracers for the presence of PAHs in disks around late type $\mathrm{T}$ Tauri stars owing to their relatively high feature/continuum ratio, and this qualitatively agrees with the fact that these two features are always present in our T Tauri stars with PAH detection. Quantitatively, Fig. 7 shows that, for the current model assumptions, the PAH features become difficult to observe for $T_{\text {eff }}<4200 \mathrm{~K}$ (later than K6) even with a relatively high PAH abundance of $\sim 6 \%$ of the dust mass in the entire disk and large flaring angles.

The observed $11.2 \mu \mathrm{m}$ line fluxes have all been rescaled to a distance of $d=150 \mathrm{pc}$, and are plotted in Fig. 9 together with the models. Also included is our typical $3 \sigma$ limit for a source at $150 \mathrm{pc}$. For comparison we added a small subsample of 12 Herbig Ae stars, selected from Acke \& van den Ancker (2004), and rescaled to 150 pc. Stars with unknown distance and/or poorly known spectral type were excluded. The observed feature/continuum ratios for the two strongest features, 6.2 and $11.2 \mu \mathrm{m}$, are compared with models in Fig. 10 .

Figure 9 shows that our template model (solid line) predicts a larger $I_{\mathrm{PAH}}$ than observed, with the exception of one source (HD 98922) for which the distance is uncertain. A lower observed $11.2 \mu \mathrm{m}$ flux can be caused by a number of conditions such as a lower PAH abundance, a smaller flaring angle or a disk orientation close to edge-on (see below). Included in Fig. 9 are two additional model runs, where the PAH abundance has been lowered by a factor of 10 (dashed line) and 100 (dash-dotted line). Most of the observations fall within the predictions of these two models. Only the model with a $100 \times$ lower PAH abundance predicts a $11.2 \mu \mathrm{m}$ feature strength for G-type stars below our 


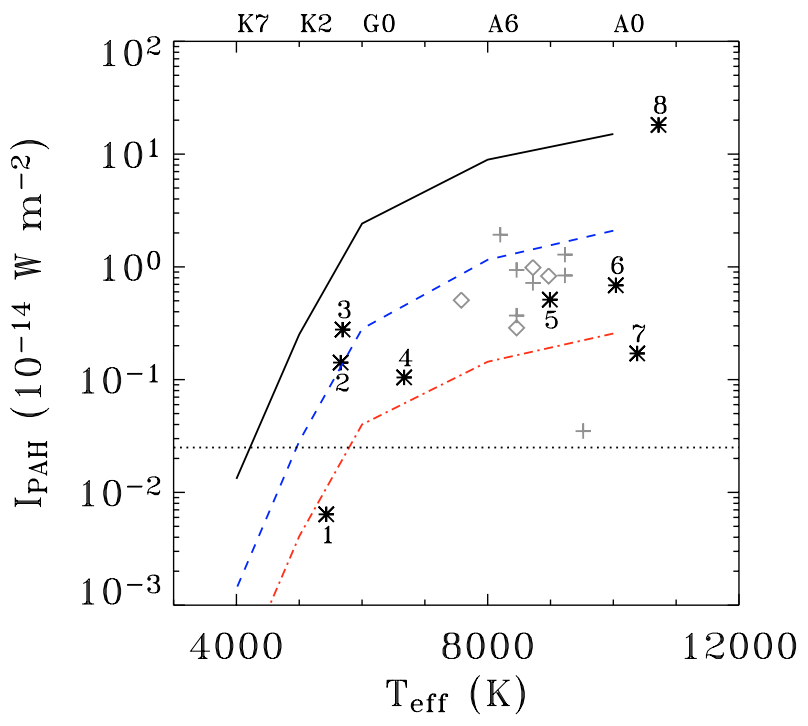

Fig. 9. Strength of $11.2 \mu \mathrm{m}$ PAH feature $I_{\mathrm{PAH}}$ (scaled to a distance of $150 \mathrm{pc}$ ) versus $T_{\text {eff }}$. Black solid line represents our template model, based on Fig. 7; dashed and dash-dotted lines represent our models with a $10 \times$ and $100 \times$ lower PAH abundance respectively. Black ' $*$ ' symbols are for $\mathrm{c} 2 \mathrm{~d}$ sources. Grey diamonds are ISO upper limits of the $11.2 \mu \mathrm{m}$ feature strength for Herbig Ae sources, grey ' + ' symbols are ISO detections. c2d sources are labelled as follows. 1: T Cha; 2: $\mathrm{LkH} \alpha 330$; 3: SR 21 N; 4: HD 135344; 5: RR Tau; 6: VV Ser; 7: HD 101412; 8: HD 98922. The dotted grey line indicates our typical $3 \sigma$ sensitivity limit of $2.5 \times 10^{-16} \mathrm{~W} \mathrm{~m}^{-2}$ for sources at $d=150 \mathrm{pc}$.

Spitzer detection limit at $d=150 \mathrm{pc}$. One source, T Cha, is detected despite the fact it falls below this formal detection limit because it has a distance of $d=66 \mathrm{pc}$.

Figure 10 indicates that the template model fits the observed $11.2 \mu \mathrm{m}$ feature/continuum ratios for the 4 low mass sources, even though it overpredicts $I_{\mathrm{PAH}}$. The model with $10 \times$ lower $\mathrm{PAH}$ abundance fits the feature/continuum ratio of three of our HAeBe stars. However both models with $10 \times$ and $100 \times$ lower (below plot limit) PAH abundance predict a feature/continuum ratio below our Spitzer detection limit for spectral types below G0. This suggests that the small number of T Tauri detections (3 out of 38) are presumably outliers, with abnormally high feature/continuum PAH features. This discrepancy will be further discussed in Sect. 5.5 and is most likely due to an abnormally low continuum in these sources.

\subsection{Additional UV radiation and relation with $\mathrm{H} \alpha$}

The models presented in Sect. 5.2 include only the stellar radiation, but not any additional sources of UV. Excess UV radiation compared with the stellar photosphere has been observed from at least some T Tauri stars (e.g., Herbig \& Goodrich 1986, Costa et al. 2000, Bergin et al. 2003). From a constructed composite FUV spectrum, derived from two K7 sources representative of low-mass T Tauri stars, Bergin et al. (2003, and references therein) find an overall FUV continuum flux at $r=100 \mathrm{AU}$ on the order of a few hundred times the Habing field. The effect of this additional UV is to shift absolute feature strengths to those appropriate for higher $T_{\text {eff }}$, close to $T_{\text {eff }} \sim 10000 \mathrm{~K}$. If a large fraction of $\mathrm{T}$ Tauri stars would have such additional UV radiation this leads to the question why not more sources were detected, since the feature strength should be more than sufficient if PAHs are present at our template abundance. This would

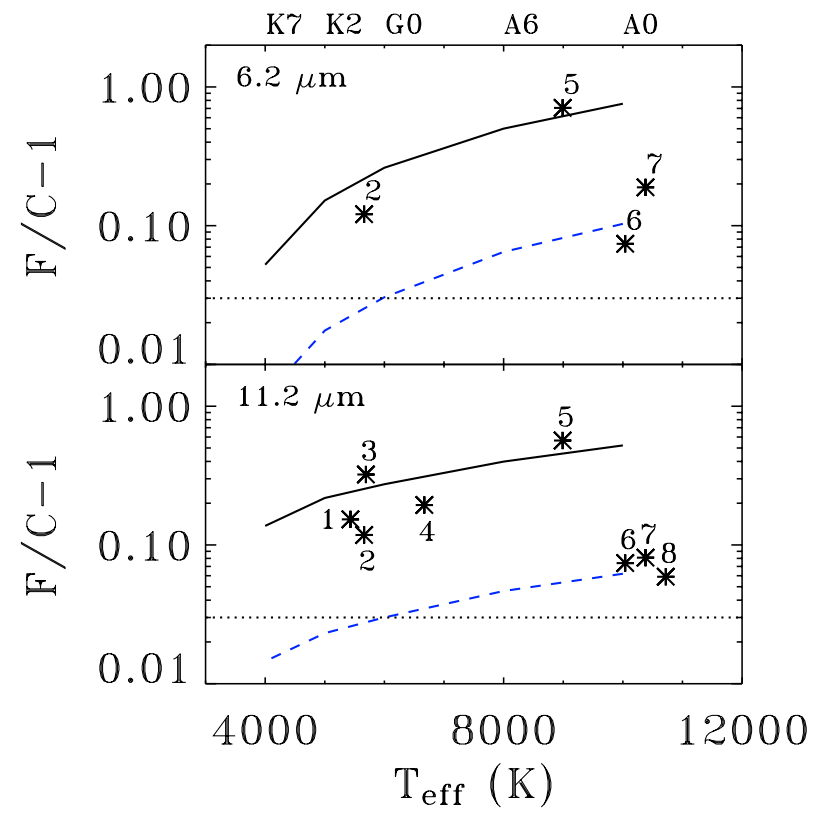

Fig. 10. Feature / continuum ratio for the 6.2 and $11.2 \mu \mathrm{m}$ PAH features; solid line represents our template model, based on Fig. 7; dashed line represents our model with a 10x lower PAH abundance.

further strengthen the evidence for low PAH abundances in a large fraction of $\mathrm{T}$ Tauri disks.

The origin of excess UV radiation from T Tauri stars is not fully clear, but is usually thought to originate from the shock in the magnetospheric accretion column associated with material falling from the inner disk onto the stellar surface. One indication of accretion activity is the strength of the $\mathrm{H} \alpha$ emission line, measured in $\mathrm{H} \alpha$ equivalent width $(E W)$ (Cabrit et al. 1990). Literature values of $\mathrm{H} \alpha E W$ measurements are listed in Table 1. In our sample, $\mathrm{LkH} \alpha 330$ is a classical T Tauri star ( $\mathrm{H} \alpha$ $E W \geq 10 \AA$ ), while $\mathrm{T}$ Cha should be classified as a weak line $\mathrm{T}$ Tauri star $(\mathrm{H} \alpha E W<10 \AA)$. We find no correlation between the $11.2 \mu \mathrm{m}$ PAH feature strength and $\mathrm{H} \alpha E W$. However, single measurements of $\mathrm{H} \alpha E W$ may not necessarily be a reliable tracer of the average accretion luminosity. As an example, for T Cha the quoted $\mathrm{H} \alpha E W$ of $2 \AA$ by Alcalá et al. (1993) is indicative of a low accretion rate, while Sylvester et al. (1996) have later classified it as a YY Orionis star with strong UV continuum emission and substantial variability on short timescales. Another problem is that not all of this UV radiation from the accretion shock may reach the disk, but can be absorbed by the overlying accretion column and/or any bi-polar outflow material (Alexander et al. 2005).

An alternative interpretation of the enhanced UV radiation is stellar activity, especially at later evolutionary stages when the accretion becomes less important. This mechanism has been used by Kamp \& Sammar (2004) to model the UV field around young G-type stars.

\subsection{PAH abundance}

The discussion in Sect. 5.1 has illustrated some of the effects of the PAH abundance on the PAH features. As seen in Figs. 9 and 10, the PAH features decrease in line flux and feature to continuum ratio with decreasing $\mathrm{PAH}$ abundance, with a similar trend for all the PAH features. Decreasing the PAH abundance also affects the overall SED, increasing the continuum radiation 
at wavelengths longer than $2 \mu \mathrm{m}$ and at $\mathrm{UV}$ wavelengths around $0.2 \mu \mathrm{m}$.

For sources with detected $11.2 \mu \mathrm{m}$ PAH feature, the line strengths indicate PAH abundances between 10 and $100 \times$ lower than the template model abundance, i.e., between 0.06 and $0.6 \%$ of dust mass. With respect to hydrogen, this translates to $\mathrm{PAH}$ abundances between $5 \times 10^{-9}$ and $5 \times 10^{-8}$. Geometry affects these numbers by at most a factor of a few (see Sect. 5.5). Thus, the absence of PAH features in the majority of our T Tauri sources may be partly caused by a lower PAH abundance than found in molecular clouds. However, this does not mean that PAHs are absent; even at these lower abundances, PAHs can still have an important influence in terms of UV opacities and heating rates in the surface layer of the disk (Jonkheid et al., submitted). For comparison, the PAH abundance inferred for the HD 141569 transitional disk is $0.00035 \%$ compared to dust (Li \& Lunine 2003 ) or $1.5 \times 10^{-10}$ with respect to hydrogen (Jonkheid et al. 2006).

\subsection{Disk geometry}

Finally, the effect of the disk geometry on the PAH features is addressed. The template disk and PAH model is used, again with $T_{\text {eff }}=10000 \mathrm{~K}$. The pressure scale height of the disk at the outer radius is varied between $H_{p} / R_{\text {out }}=0.06$ and 0.22 , while the pressure scale height of the inner rim is kept constant at $H_{p} / R_{\text {in }}=0.02$. This illustrates the effect on the PAH features of a flaring disk versus a flatter disk. SEDs for varying values of the scale height of the outer disk are shown in Fig. 11. The line flux of the PAH features is found to slowly decrease as the disk becomes less flared by lowering the outer disk scale height from $H / R_{\text {out }}=0.22$ to 0.06 . The feature over continuum ratio is found to increase slightly with decreasing $H / R_{\text {out }}$ for most features, with the exception of the 3.3 and $6.2 \mu \mathrm{m}$ features, where the feature/continuum ratio decreases for $H / R_{\text {out }}<0.1$. This trend is caused by a more rapid decrease of the silicate continuum flux compared to the PAH peak flux. All the main PAH features can be distinguished for the flaring disk, although the $8.6 \mu \mathrm{m}$ feature has a low feature to continuum ratio of $\sim 3-10 \%$.

To compare this scenario to our PAH detections, a measure for the flaring index of the disk is taken to be the shape of the SED, obtained by dividing the observed $\nu F_{v}$ at $35 \mu \mathrm{m}$ over $13 \mu \mathrm{m}$ (Kessler-Silacci et al. 2006). This ratio is compared to the measured $11.2 \mu \mathrm{m}$ line flux. No clear evidence for a correlation between the $11.2 \mu \mathrm{m}$ line strength and a rising/non-rising SED is found. Together with the lack of strong changes in Fig. 11, this suggests that disk flaring alone is not sufficient to explain the PAH non-detections in our sample.

A second geometry effect, disk thickness, can have a direct effect on the PAH feature strength $I_{\mathrm{PAH}}$. A geometrically thicker disk captures a larger portion of the stellar radiation field than a flatter disk, and hence the response in both the dust continuum and the PAH features is stronger. Observations of spectral energy distributions of disks around pre-main-sequence stars have indicated that disk geometries vary strongly from source to source (Meeus et al. 2001), and that this factor can therefore potentially affect the spectrum. Test model runs show that increasing the disk geometrical thickness by a factor 2, by increasing the pressure scale height $H_{p}$ throughout the entire disk, introduces an increase in $I_{\mathrm{PAH}}$ of $\sim 1.5-2$, while the ratio of PAH feature over continuum ratio decreases by a factor $\sim 1.4-1.8$. The disk would have to be a factor $\sim 60-80$ thinner for the PAH features to have been undetectable for $T_{\mathrm{eff}}<6000 \mathrm{~K}(\mathrm{G} 0)$, for the template abundance.
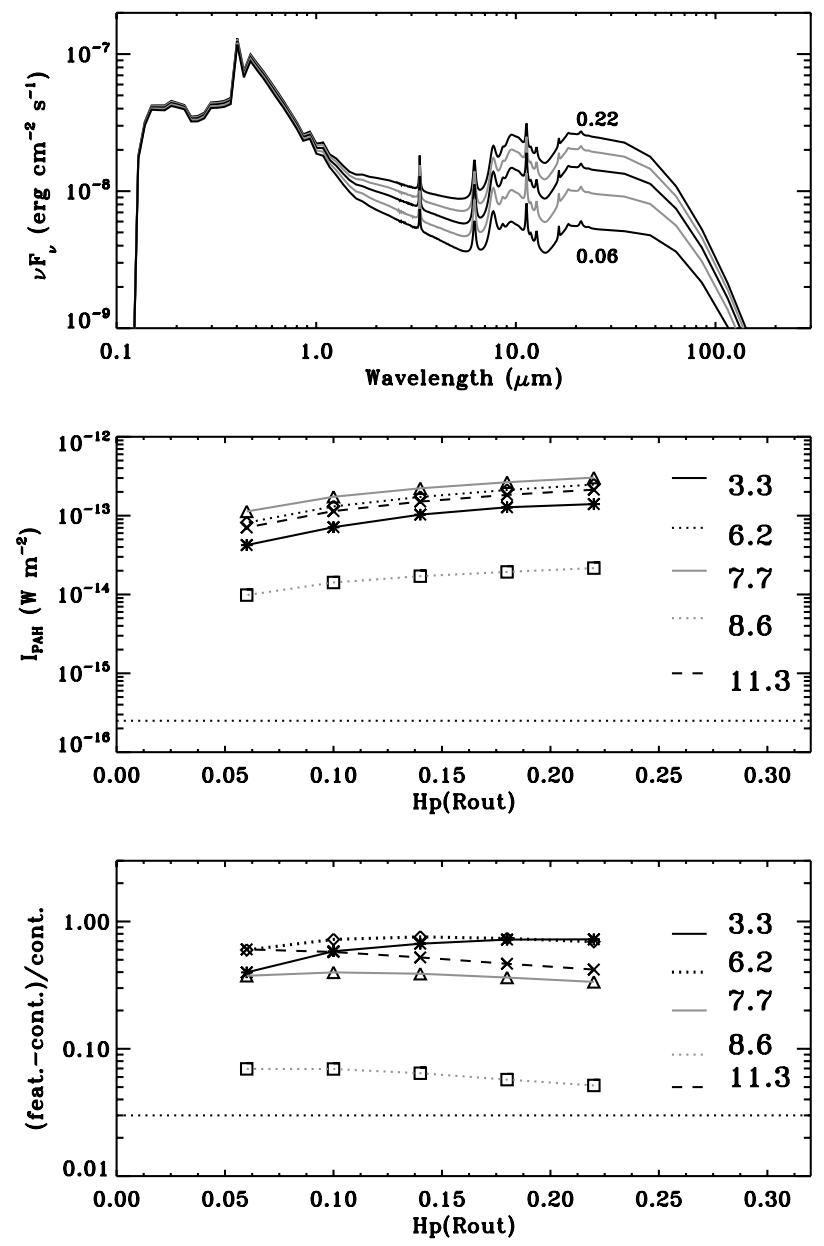

Fig. 11. Top panel: Model SEDs of a star+disk with PAH, with scale height of outer disk $H / R_{\text {out }}=0.06,0.1,0.14,0.18$ and 0.22 at a distance of $150 \mathrm{pc}$. Middle panel: PAH line fluxes for the corresponding models. Bottom panel: Feature to continuum ratio of the various features. The dotted line indicates our observational limits.

A third geometry effect, inclination, affects both $I_{\mathrm{PAH}}$ and the feature over continuum ratio. Our template models are run for face-on disks $\left(i=5^{\circ}\right)$. When the template model is observed at angles up to $i \sim 60^{\circ}, I_{\mathrm{PAH}}$ remains relatively unchanged $(<3 \%)$ and the feature over continuum increases by about $10 \%$. For an almost edge-on disk $\left(i=80^{\circ}\right), I_{\mathrm{PAH}}$ has decreased by $\sim 40 \%$, while the feature over continuum is a factor $\sim 4$ larger. Inclination may be an explanation for some of the sources with abnormally large $\mathrm{F} / \mathrm{C}$ ratio, compared with their feature strength, e.g. RR Tau.

A fourth geometry effect which could explain an unusually high feature/continuum ratio is the introduction of a large scale gap in the inner dust disk, for example as the result of dust coagulation or possibly through clearing by one or more planet(s), although the latter would require extreme circumstances. This lowers the $3-13 \mu \mathrm{m}$ continuum flux while keeping the PAH feature strength the same. The motivation for this explanation comes from the SED of the small fraction of T Tauri stars in our sample that are detected, all of which have a higher $11.2 \mu \mathrm{m}$ feature/continuum ratio than would be consistent with their $11.2 \mu \mathrm{m}$ fluxes within the standard model. The SEDs of these 4 detected sources show remarkably low emission at $10 \mu \mathrm{m}$ followed by a steep rise towards $20 \mu \mathrm{m}$, suggestive of a gap opening up in the dust disk (Brown et al., in prep.). Examples of similar SEDs include those of DM Tau, GM Aur and CoKu Tau/4 (e.g. 
D'Alessio et al. 2005), for which the continuum flux around $11 \mu \mathrm{m}$ is also depressed by more than an order of magnitude compared with that of a standard T Tauri star in Taurus.

\subsection{Model summary}

In summary, all three effects of reducing the stellar temperature (and thus the amount of optical and UV radiation), reducing the PAH abundance, and decreasing the flaring angle or thickness of the disk are shown to decrease the absolute strength of the PAH features, as expected. The feature over continuum ratio trends are similar for the 6.2, 7.7, 8.6 and 11.2 $\mu \mathrm{m}$ PAH features. That of the $8.6 \mu \mathrm{m}$ is the lowest in all models, with typical values of $<6-10 \%$ even for models which maximize the features, which can qualitatively explain the observed lack of this feature.

The $11.2 \mu \mathrm{m}$ feature remains one of the strongest features in all models explored and this is qualitatively consistent with our observations. For the template model parameters which maximize the PAH features, PAHs are observable within the mean sensitivity limit of our observations for $T_{\text {eff }} \geq 4200 \mathrm{~K}$ (K6). For a $10 \times$ lower PAH abundance, the features are still above our sensitivity limit for $T_{\text {eff }} \geq 4900 \mathrm{~K}$ (K2), but now the feature/continuum ratio becomes the limiting factor, with values $\lesssim 5 \%$ for $T_{\text {eff }} \leq 6000 \mathrm{~K}(\mathrm{G} 0)$. This partly explains the absence of any PAH features in the spectra of more than half of the sources in the sample considered, which consists mostly of late $\mathrm{K}$ and $\mathrm{M}$ type stars.

\section{Conclusions and future work}

Spitzer-IRS spectra were obtained for a set of 54 pre-main sequence stars with disks, including $38 \mathrm{~T}$ Tauri stars and 7 Herbig Ae stars. The observations are an order of magnitude more sensitive than those used in previous surveys of PAHs in disks. We detect PAH features in at least $3 \mathrm{~T}$ Tauri stars, with an additional 14 tentative detections to be confirmed, resulting in a lower limit to the PAH detection rate in T Tauri stars of $8 \%$. Spitzer SL observations are needed to confirm the presence of the PAH features for sources where we currently only see the $11.2 \mu \mathrm{m}$ feature in the SH spectrum. All 4 sources that show hints for inner holes in their dust disk also a show clear 11.2 $\mu \mathrm{m}$ PAH feature.

The lowest mass source with PAH emission in our sample is T Cha with spectral type G8. The derived $11.2 \mu \mathrm{m}$ line intensities are between a few $\times 10^{-15}$ and $3.3 \times 10^{-16} \mathrm{~W} \mathrm{~m}^{-2}$, which is typically an order of magnitude lower than what was observed for HAeBe stars with ISO.

Radiative transfer modeling of disks coupled with PAH emission models shows that for stars of late spectral type, the $11.2 \mu \mathrm{m}$ feature is expected to be the best tracer of the presence of PAHs. The models also show that the 7.7 and $8.6 \mu \mathrm{m} \mathrm{PAH}$ features are most affected by veiling by the continuum due to strongly rising silicate emission, resulting in low feature over continuum ratios.

For the small number of $\mathrm{T}$ Tauri sources detected as well as for the Herbig stars, the measured PAH line fluxes and feature/continuum ratios are lower than those found from our template disk model which maximizes the PAH emission. Variations of the model parameters indicate that the most likely explanation is lower PAH abundances by factors of 10-100, with geometry affecting these conclusions at the level of a factor of a few. The high feature/continuum ratios for the detected $\mathrm{T}$ Tauri stars are due to their abnormally low continuum at $11 \mu \mathrm{m}$ caused by the dust holes in their inner disks.
The template model predictions indicate that the $11.2 \mu \mathrm{m}$ feature strength becomes undetectable at our sensitivity limit when $T_{\text {eff }}<4200 \mathrm{~K}$ (K6). This likely explains the absence of PAH features for the majority of sources in our sample which have spectral types later than $\mathrm{K} 0(\sim 70 \%)$. If a large fraction of these sources would have excess UV radiation at the level detected for some K7 stars, then the absence of PAH features implies a lower PAH abundance by at least an order of magnitude. The same conclusion holds for the 11 out of 38 sources with spectral types earlier than K6 which are not detected. Geometry affects these conclusions by a factor of a few. Thus, the lack of PAH detections does not mean that PAHs are absent in these disks; even at lower abundances they do need to be considered since they can affect disk structure, chemistry and gas heating.

In a follow-up paper, the combination of 5-35 $\mu \mathrm{m}$ spectra for the entire sample with IRAC and MIPS photometry will be used to improve the uncertainty in the PAH detection rate and to compare the PAH sources in terms of their disk SEDs and interpret this with the disk modeling.

Ground-based spectra using instruments like VLT-ISAAC and VLT-VISIR on 8-m class telescopes will be able to both search for presence of the $3.3 \mu \mathrm{m}$ PAH feature as well as better characterize the shape of the 8.6 and $11.2 \mu \mathrm{m}$ PAH feature through higher spectral resolution. Through higher spatial resolution, they will also permit us to put a stronger constraint on the spatial extent of the PAH features from T Tauri disks.

Acknowledgements. Support for this work, part of the Spitzer Legacy Science Program, was provided by NASA through contracts 1224608, 1230779 and 1256316 issued by the Jet Propulsion Laboratory, California Institute of Technology, under NASA contract 1407. Astrochemistry in Leiden is supported by a NWO Spinoza grant and a NOVA grant, and by the European Research Training Network "The Origin of Planetary Systems" (PLANETS, contract number HPRN-CT-2002-00308). B. Merín acknowledges funding from the "Fundación Ramón Areces (Spain). The authors wish to thank Louis Allamandola and Andrew Mattioda for discussions and new opacities and Emilie Habart for comparisons with her models.

\section{References}

Acke, B., \& van den Ancker, M. E. 2004, A\&A, 426, 151

Acke, B., van den Ancker, M. E., \& Dullemond, C. P. 2005, A\&A, 436, 209

Alcalá, J. M., Covino, E., Franchini, M., et al. 1993, A\&A, 272, 225

Alcala, J. M., Krautter, J., Schmitt, J. H. M. M., et al. 1995, A\&AS, 114, 109 Alexander, R. D., Clarke, C. J., \& Pringle, J. E. 2005, MNRAS, 358, 283

Bell, K. R., Cassen, P. M., Klahr, H. H., \& Henning, T. 1997, ApJ, 486, 372

Bergin, E., Calvet, N., D’Alessio, P., \& Herczeg, G. J. 2003, ApJ, 591, L159

Bergin, E., Calvet, N., Sitko, M. L., et al. 2004, ApJ, 614, L133

Cabrit, S., Edwards, S., Strom, S. E., \& Strom, K. M. 1990, ApJ, 354, 687

Cesarsky, D., Lequeux, J., Ryter, C., \& Gérin, M. 2000, A\&A, 354, L87

Cohen, M., \& Kuhi, L. V. 1979, ApJS, 41, 743

D’Alessio, P., Canto, J., Calvet, N., \& Lizano, S. 1998, ApJ, 500, 411

D'Alessio, P., Hartmann, L., Calvet, N., et al. 2005, ApJ, 621, 461

de Geus, E. J., de Zeeuw, P. T., \& Lub, J. 1989, A\&A, 216, 44

Desert, F.-X., Boulanger, F., \& Puget, J. L. 1990, A\&A, 237, 215

Dominik, C., Dullemond, C. P., Waters, L. B. F. M., \& Walch, S. 2003, A\&A, 398,607

Draine, B. T., \& Li, A. 2001, ApJ, 551, 807

Dullemond, C. P., \& Dominik, C. 2004, A\&A, 417, 159

Dullemond, C. P., Dominik, C., \& Natta, A. 2001, ApJ, 560, 957

Dullemond, C. P., van Zadelhoff, G. J., \& Natta, A. 2002, A\&A, 389, 464

Dunkin, S. K., Barlow, M. J., \& Ryan, S. G. 1997, MNRAS, 286, 604

Evans, N. J., Allen, L. E., Blake, G. A., et al. 2003, PASP, 115, 965

Fernandez, M., Ortiz, E., Eiroa, C., \& Miranda, L. F. 1995, A\&AS, 114, 439

Finkenzeller, U., \& Mundt, R. 1984, A\&AS, 55, 109

Geers, V. C., Augereau, J.-C., Pontoppidan, K. M., et al. 2005, High resolution infrared spectroscopy in astronomy, ed. H. U. Kaeufl, R. Siebenmorgen, and A.F.M. Moorwood. ESO astrophysics symposia (Berlin: Springer), 239

Gray, R. O., \& Corbally, C. J. 1994, AJ, 107, 742

Guhathakurta, P., \& Draine, B. T. 1989, ApJ, 345, 230

Habart, E., Natta, A., \& Krügel, E. 2004, A\&A, 427, 179 
Habart, E., Natta, A., Testi, L., \& Carbillet, M. 2005, ArXiv Astrophysics e-prints

Habart, E., Verstraete, L., Boulanger, F., et al. 2001, A\&A, 373, 702

Habing, H. J. 1968, Bull. Astron. Inst. Netherlands, 19, 421

Hernández, J., Calvet, N., Briceño, C., Hartmann, L., \& Berlind, P. 2004, AJ, 127,1682

Hony, S., Van Kerckhoven, C., Peeters, E., et al. 2001, A\&A, 370, 1030

Houck, J. R., Roellig, T. L., van Cleve, J., et al. 2004, ApJS, 154, 18

Houk, N. 1978, Michigan catalogue of two-dimensional spectral types for the HD stars (Ann Arbor : Dept. of Astronomy, University of Michigan)

Jonkheid, B., Faas, F. G. A., van Zadelhoff, G.-J., \& van Dishoeck, E. F. 2004, A\&A, 428, 511

Jonkheid, B., Kamp, I., Augereau, J.-C., \& van Dishoeck, E. F. 2006, A\&A, 453, 163

Kamp, I., \& Dullemond, C. P. 2004, ApJ, 615, 991

Kamp, I., \& Sammar, F. 2004, A\&A, 427, 561

Kenyon, S. J., Dobrzycka, D., \& Hartmann, L. 1994, AJ, 108, 1872

Kessler-Silacci, J., Augereau, J.-C., Dullemond, C. P., et al. 2006, ApJ, 639, 275

Kessler-Silacci, J. E., Hillenbrand, L. A., Blake, G. A., \& Meyer, M. R. 2005, ApJ, 622, 404

Li, A., \& Draine, B. T. 2001, ApJ, 554, 778

Li, A., \& Lunine, J. I. 2003, ApJ, 594, 987

Manske, V., \& Henning, T. 1999, A\&A, 349, 907

Martin, E. L., Montmerle, T., Gregorio-Hetem, J., \& Casanova, S. 1998, MNRAS, 300, 733
Mattioda, A. L., Hudgins, D. M., \& Allamandola, L. J. 2005, ApJ, 629, 1188 Meeus, G., Waters, L. B. F. M., Bouwman, J., et al. 2001, A\&A, 365, 476 Mora, A., Merín, B., Solano, E., et al. 2001, A\&A, 378, 116

Pascucci, I., Wolf, S., Steinacker, J., et al. 2004, A\&A, 417, 793

Pech, C., Joblin, C., \& Boissel, P. 2002, A\&A, 388, 639

Peeters, E., Hony, S., Van Kerckhoven, C., et al. 2002, A\&A, 390, 1089 Peeters, E., Spoon, H. W. W., \& Tielens, A. G. G. M. 2004, ApJ, 613, 986 Prato, L., Greene, T. P., \& Simon, M. 2003, ApJ, 584, 853

Przygodda, F., van Boekel, R., Àbrahàm, P., et al. 2003, A\&A, 412, L43

Siebenmorgen, R., Kruegel, E., \& Mathis, J. S. 1992, A\&A, 266, 501

Siess, L., Dufour, E., \& Forestini, M. 2000, A\&A, 358, 593

Sloan, G. C., Keller, L. D., Forrest, W. J., et al. 2005, ApJ, 632, 956

Spoon, H. W. W., Keane, J. V., Tielens, A. G. G. M., et al. 2002, A\&A, 385, 1022

Straizys, V., Cernis, K., \& Bartasiute, S. 1996, Baltic Astron., 5, 125

Sylvester, R. J., Skinner, C. J., Barlow, M. J., \& Mannings, V. 1996, MNRAS, 279,915

Thé, P. S., de Winter, D., \& Perez, M. R. 1994, A\&AS, 104, 315

Tielens, A. G. G. M., \& Hollenbach, D. 1985, ApJ, 291, 722

van Boekel, R., Waters, L. B. F. M., Dominik, C., et al. 2003, A\&A, 400, L21

van Boekel, R., Waters, L. B. F. M., Dominik, C., et al. 2004, A\&A, 418, 177

van den Ancker, M. E., de Winter, D., \& Tjin A Djie, H. R. E. 1998, A\&A, 330, 145

van Diedenhoven, B., Peeters, E., Van Kerckhoven, C., et al. 2004, ApJ, 611, 928

Van Kerckhoven, C., Hony, S., Peeters, E., et al. 2000, A\&A, 357, 1013

Werner, M. W., Roellig, T. L., Low, F. J., et al. 2004, ApJS, 154, 1 
V. C. Geers et al.: PAH emission features from disks, Online Material $p 1$

\section{Online Material}




\section{Appendix A: Model tests and comparison with Habart et al. 2004}

There are various differences in the modeling approach between the Habart et al. (2004) paper and our models, which are summarized in Table A.1. First, the H04 model is a 1+1D model, meaning that the model consists of a series of vertical 1D models at different radii, combined to make a full disk structure. This method follows the irradiation-angle philosophy used in many other models as well, e.g. D'Alessio et al. (1998), Bell et al. (1997), Dullemond et al. (2002). In our model we use full 3D radiative transfer based on an axisymmetric disk density structure. Our model therefore also includes emission from the dust inner rim, contrary to the approach of H04. Our 3D approach allows radiative energy to be exchanged between adjacent radii, which is not possible in the $1+1 \mathrm{D}$ approach. Consequently the model SEDs differ somewhat between these two types of models. Specifically, for a flaring disk, the radiation will more readily escape in the polar direction than in the radial direction, because of the larger optical depth in the latter direction. When the disk is viewed face-on, the SED will be boosted in the 3D approach compared to the $1+1 \mathrm{D}$ approach by up to $\sim 40 \%$. Moreover, the axisymmetric 3D models described here allow the treatment of radiative transfer in disk geometries that are not flaring, e.g. selfshadowed disks (see Sect. 5 for examples). On the other hand, in our models the vertical geometric thickness of the disk is only estimated to be roughly consistent with hydrostatic equilibrium, whereas the H04 model includes detailed hydrostatic equilibrium. The results of these two approaches, however, are nearly identical.

There are also fundamental differences in the opacities and the treatment of dust grains in the radiative transfer. Most importantly, H04 thermally decouple the carbon grains from the silicate grains, while we have both grain types at the same temperature and mix the opacities into a single opacity. Our assumption may be more realistic because silicate and carbon grains can only have different temperatures at the same location in the disk if such grains are physically disconnected. Only a small amount of coagulation is required to get these grains in physical contact, forcing them to have the same temperature. The result is that the silicate feature strength over the continuum is much stronger in our case than in the case of H04, as can be seen in their Fig. 3 as compared to our Fig. 8. If the graphite is decoupled from the silicate, the graphite heats up and the silicate cools down due to the much lower optical/NIR opacity of silicate compared with graphite. The superheated graphite emission therefore fills in the spectrum on both sides of the silicate feature in the case of thermally decoupled grains.

For stars with lower effective temperature, radiation at longer wavelengths becomes more inportant. Our models include the new PAH opacities of Mattioda et al. (2005) at optical and nearinfrared wavelengths, which are higher than those adopted by H04. In our model we do not include PAH-destruction in the inner disk, in contrast with H04. Test models with and without PAH destruction show only small differences, however.

The biggest differences between $\mathrm{H} 04$ and our models are likely due to the treatment of the stellar radiation. Our models assume that the full stellar flux can irradiate the disk, whereas $\mathrm{H} 04$ assume that the disk goes all the way to the stellar surface and occults the lower half of the star. Hence the H04 models have only half the illuminating flux as ours. Moreover, they include the star as a blackbody emitter with main sequence stellar parameters, while in our model we use a Kurucz model with premain sequence values.

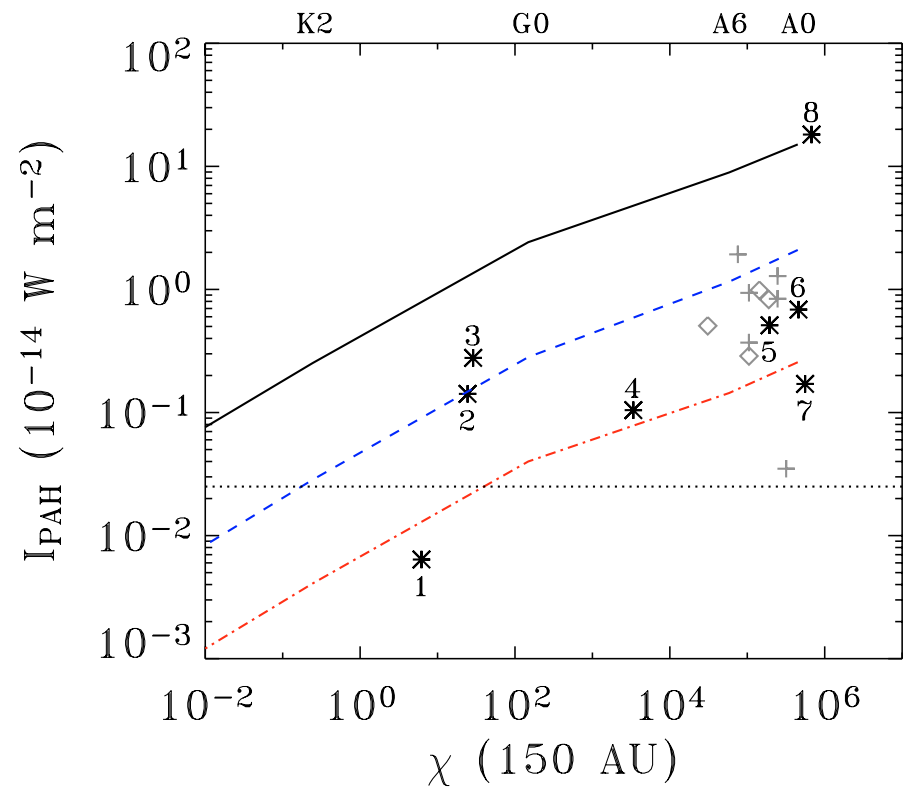

Fig. A.1. Strength of $11.2 \mu \mathrm{m}$ PAH feature $I_{\mathrm{PAH}}$ (scaled to a distance of $150 \mathrm{pc}$ ) versus $\chi$, the integrated FUV field $(6-13.6 \mathrm{eV})$ for a ZAMS star, after Habart et al. (2004, their Fig. 8). Solid line represents our template model, based on Fig. 7; dashed and dash-dotted lines represent our models with a 10x and 100x lower PAH abundance respectively. Black '*' symbols are for c2d sources. Grey diamonds are ISO upper limits of the $11.2 \mu \mathrm{m}$ feature strength for Herbig Ae sources, grey '+' symbols are ISO detections. c2d sources are labelled as follows. 1: T Cha; 2: LkH $\alpha$ 330; 3: SR 21 N; 4: HD 135344; 5: RR Tau; 6: VV Ser; 7: HD 101412; 8: HD 98922 . The dotted grey line indicates our typical $3 \sigma$ sensitivity limit of $2.5 \times 10^{-16} \mathrm{~W} \mathrm{~m}^{-2}$ for sources at $d=150 \mathrm{pc}$.

To quantitatively compare the two models, we have plotted our PAH intensities in the same way as H04, as functions of the integrated stellar radiation field in the FUV (6$13.6 \mathrm{eV}, 912-2050 \AA$ ) wavelength range. The UV flux is usually specified relative to the average interstellar radiation field from Habing (1968) integrated over this wavelength range, which is $1.6 \times 10^{-3} \mathrm{erg} \mathrm{cm}^{-2} \mathrm{~s}^{-1}$ (Tielens \& Hollenbach 1985). This quantity is most often referred to as $G_{0}$ but we refer to it here as $\chi$ to be consistent with $\mathrm{H} 04$. H04 assume a single blackbody for the stellar radiation field for stars of spectral type B5-G0 and rescale the flux from the stellar surface $R_{*}$, to the flux at a distance from the star of $150 \mathrm{AU}$, as a reference point in the middle of their disk.

We have calculate $\chi$ for our models as presented in Fig. 9 using Kurucz spectra and pre-main sequence parameters. The results are presented in Fig. A.1. Moreover, we have run our template model assuming a blackbody stellar spectrum and mainsequence stellar parameters, with $T_{\text {eff }}=15000,12300,10500$, $8500,7000,6000$ and $5000 \mathrm{~K}$, corresponding to spectral types B4, B7, A0, A3, F2, F9 and K2, respectively (Gray \& Corbally 1994). Fig. A.2 shows the results for the template PAH abundance (same as H04, dark solid line) and 10× lower (dashed line) and includes the model prediction by H04 (light solid line). The observational data are included in both figures, with $\chi$ computed from the spectral types in the same way as described above for the pre-main sequence stars.

It is seen from Figs. A. 1 and A. 2 that for the same PAH abundance, our models predict factors of 4-5 stronger PAH features than the H04 models. In fact, our entire SED (continuum and features) is stronger than in their models. This means that our model disk absorbs more stellar radiation and hence re-emits 
Table A.1. List of differences between the model presented here and that of Habart et al. (2004).

\begin{tabular}{lll}
\hline \hline Model aspect & This paper & H04 \\
\hline Radiative transfer & Axisymmetric 3D & $1+1 \mathrm{D}$ \\
Disk structure & Inner rim & - \\
$\rho(Z)$ & Parametrized & Self-consistent \\
Stellar spectrum & Kurucz & BB \\
Stellar parameters & PMS & MS \\
Stellar flux & $100 \%$ & $50 \%$ \\
PAH evaporation & $1 / 2$ & yes \\
$T-$ decoupling & no & yes \\
Optical-IR PAH opacities & Li \& Draine (2001), & Li \& Draine (2001), \\
& Mattioda et al. (2005) & Desert et al. (1990) \\
\hline
\end{tabular}

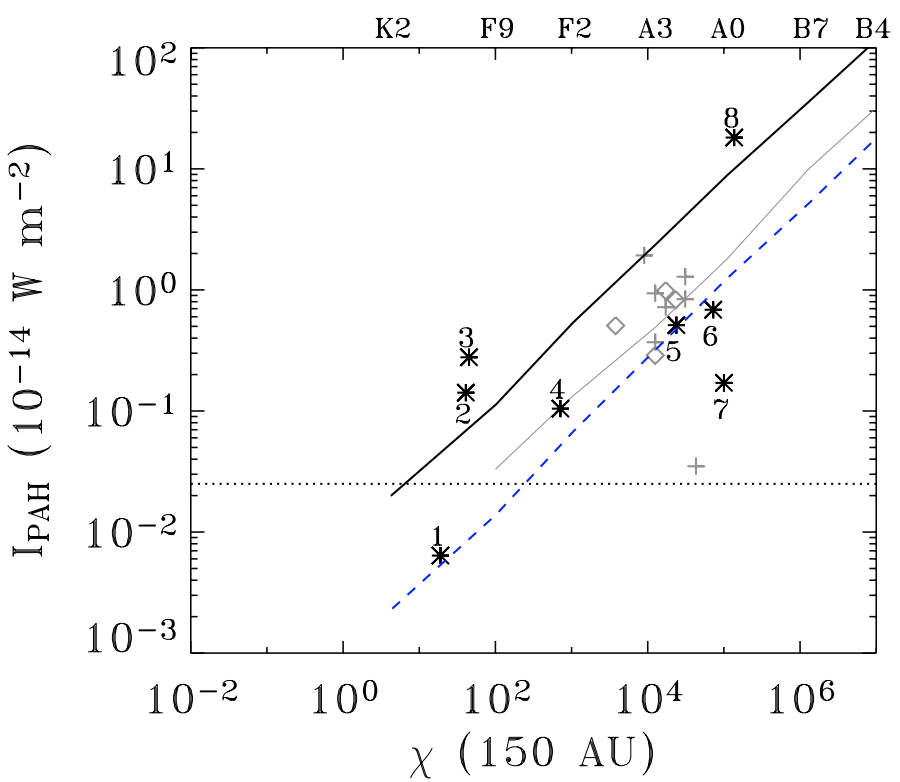

Fig. A.2. As Fig. A.1, but assuming a blackbody stellar spectrum and main-sequence stellar parameters. Dark solid line represents our template model, dashed line our model with a $10 \times$ lower PAH abundance. Light solid line represents the H04 disk model results for the $11.2 \mu \mathrm{m}$ feature.

more radiation in the infrared, both in PAHs and in thermal continuum. A factor of two can be traced back to the different assumption for the irradiative flux, whereas another $\sim 40 \%$ is due to the "boosting" effect in the $3 \mathrm{D}$ versus $1+1 \mathrm{D}$ approach in this near-face-on geometry. The remaining factor of $\sim 2$ is ascribed to the other differences described above in terms of disk structure, geometry, treatment of dust grains, PAH destruction and PAH opacities. Another difference is that our template model assumes a mix of neutral and ionized PAHs whereas that of $\mathrm{H} 04$ assumes only neutral PAHs. A comparison of feature-over-continuum ratios, in which many of these effects drop out, would be instructive but H04 do not quote such values.

Comparison of Figs. A.1 and A.2 shows that the $11.2 \mu \mathrm{m}$ fluxes are lower if blackbody radiation is assumed with main sequence stellar parameters, especially for T Tauri stars (later than F8). Both our blackbody models with $10 \times$ lower abundance as well as the H04 models fit most of the ISO and Spitzer detections for HAeBe stars. Both models predict that for T Tauri stars of spectral type G5 and later, $I_{\mathrm{PAH}}$ to falls below our Spitzer detection limit for $d=150 \mathrm{pc}$. However, the H04 models underpredict the detected $11.2 \mu \mathrm{m}$ strengths of some of the lowest luminosity sources in our sample, whereas our models with standard PAH abundance come much closer. In general, the PAH emission from $T$ Tauri stars, when detected, appear better modeled with Kurucz spectra for pre-main sequence stars than with blackbody spectra for main sequence parameters. Overall, the main conclusions from our paper are not affected qualitatively if the H04 models with blackbodies were used to interpret the data. Quantitatively, the threshold for detection shifts to earlier spectral types (G5 versus K5) and the inferred PAH abundances are factors of 4-5 higher.

Finally, we note that a multitude of tests of our code were performed in addition to the tests that the RADMC code already has gone through (e.g. Pascucci et al. 2004). We tested that our model conserves luminosity; we compared the SED without PAHs to the SED produced by the Chiang \& Goldreich-type model described in Dullemond et al. (2001); we estimated what the SED strength should be on the basis of the disk geometric thickness; and we performed optically thin models consisting purely of PAH grains and compared to the models of Li \& Draine (2001). All these tests confirmed the validity of the models.

\section{Appendix B}

Figures B.1 and B.2 present blow-ups of the 11.2 and $12.8 \mu \mathrm{m}$ PAH features. 
V. C. Geers et al.: PAH emission features from disks, Online Material $p 4$
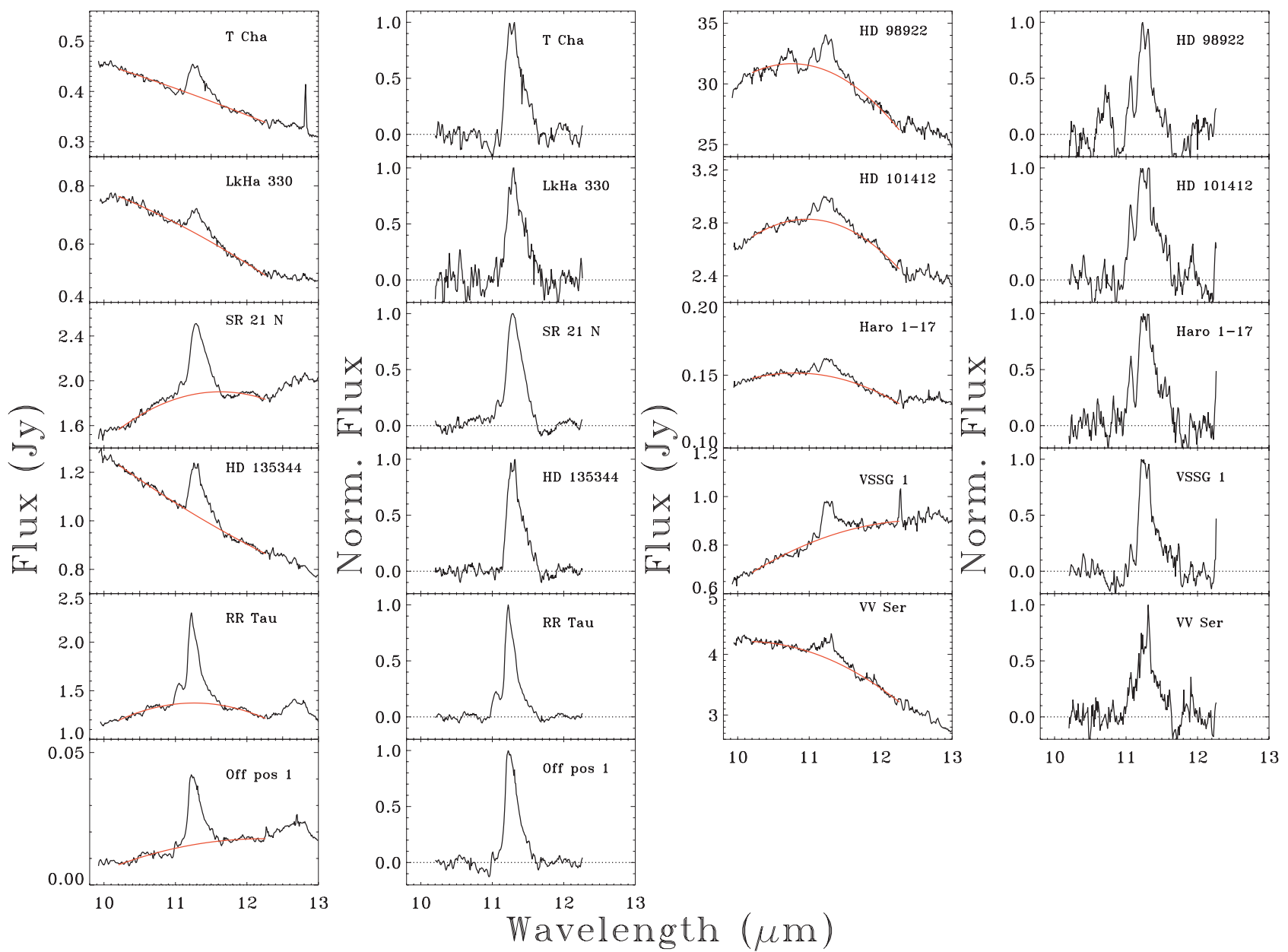

Fig. B.1. Left: Blow-up of the Spitzer IRS high resolution spectra around the $11.2 \mu \mathrm{m}$ PAH feature. A simple fit of the continuum flux below the PAH feature is plotted with a dotted line. Right: Continuum subtracted spectra, normalised to the peak flux of the PAH feature. 
V. C. Geers et al.: PAH emission features from disks, Online Material p 5
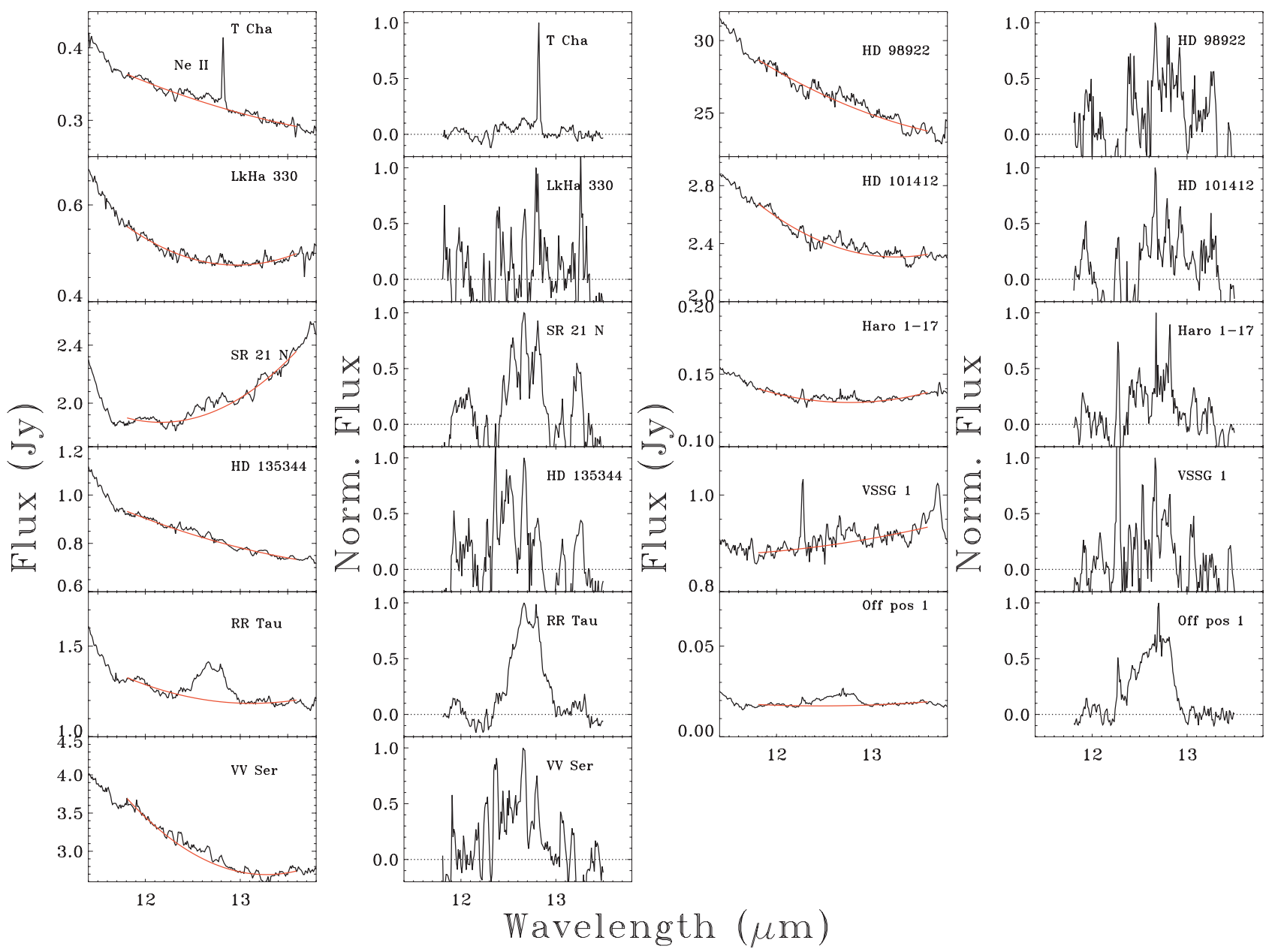

Fig. B.2. Left: Blow-up of the Spitzer IRS high resolution spectra around the $12.8 \mu \mathrm{m}$ PAH feature. A simple fit of the continuum flux below the PAH feature is plotted with a dotted line. Right: Continuum subtracted spectra, normalised to the peak flux of the PAH feature. 